\title{
Constraining Higgs mediated dark matter interactions
}

\author{
Admir Greljo, ${ }^{a}$ J. Julio, ${ }^{a}$ Jernej F. Kamenik, ${ }^{a, b}$ Christopher Smith ${ }^{c}$ and Jure Zupan ${ }^{d}$ \\ ${ }^{a}$ J. Stefan Institute, \\ Jamova 39, P.O. Box 3000, 1001 Ljubljana, Slovenia \\ ${ }^{b}$ Department of Physics, University of Ljubljana, \\ Jadranska 19, 1000 Ljubljana, Slovenia \\ ${ }^{c}$ LPSC, Université Joseph Fourier Grenoble 1, CNRS/IN2P3 UMR5821, \\ Institut Polytechnique de Grenoble, \\ 53 rue des Martyrs, 38026 Grenoble Cedex, France \\ ${ }^{d}$ Department of Physics, University of Cincinnati, \\ Cincinnati, Ohio 45221, U.S.A. \\ E-mail: admir.greljo@ijs.si, julio@ijs.si, jernej.kamenik@ijs.si, \\ chsmith@lpsc.in2p3.fr, zupanje@ucmail.uc.edu
}

ABSTRACT: We perform an analysis of Higgs portal models of dark matter (DM), where DM is light enough to contribute to invisible Higgs decays. Using effective field theory we show that DM can be a thermal relic only if there are additional light particles present with masses below a few $100 \mathrm{GeV}$. We give three concrete examples of viable Higgs portal models of light DM: (i) the SM extended by DM scalar along with an electroweak triplet and a singlet, (ii) a Two Higgs Doublet Model of type II with additional scalar DM, (iii) SM with DM and an extra scalar singlet that is lighter than DM. In all three examples the $\mathcal{B}(h \rightarrow$ invisible) constraint is not too restrictive, because it is governed by different parameters than the relic abundance. Additional light particles can have implications for flavor violation and collider searches.

Keywords: Higgs Physics, Beyond Standard Model, Cosmology of Theories beyond the $\mathrm{SM}$

ARXiv EPRINT: 1309.3561 


\section{Contents}

1 Introduction 1

2 Higgs portals in effective field theory 3

3 Suppressed Higgs decays to dark sector 4

4 Examples of viable Higgs portal models $\quad 11$

$\begin{array}{lll}\text { 4.1 } & \mathrm{SM}+\mathrm{DM} \text { with an extra triplet and a singlet } & 11\end{array}$

$\begin{array}{lll}4.2 & \text { 2HDM-II + DM } & 13\end{array}$

$\begin{array}{lll}\text { 4.3 } & \mathrm{SM}+\mathrm{DM} \text { with extra scalar singlet } & 18\end{array}$

5 Conclusions $\quad 20$

$\begin{array}{ll}\text { A Relic density and direct detection } & 21\end{array}$

B Analysis of Higgs data 23

\section{Introduction}

The narrow resonance with mass $m_{h} \simeq 125 \mathrm{GeV}$ that was recently discovered at the LHC [1, 2] is a scalar [3-7] and has interactions consistent with those of the standard model (SM) Higgs boson [8, 9]. At present the experimental uncertainties are still relatively large and even $\mathcal{O}(1)$ deviations with respect to the SM couplings are possible. One of the more intriguing possibilities is that the Higgs could couple to dark matter (DM).

The argument in favor of this possibility is quite general. Assuming that the discovered scalar is part of the Higgs electroweak doublet $H$, then $H^{\dagger} H$ is the only gauge and Lorentz invariant relevant operator in the SM. As such it can act as the "Higgs portal" to DM [10]. The experimental searches place a number of nontrivial constraints on this idea. A pivotal parameter in the constraints is the DM mass. If DM is light, $m_{\mathrm{DM}}<m_{h} / 2$, then Higgs can decay into DM. The resulting invisible decay width of the Higgs is bounded at $95 \%$ CL to $\mathcal{B}(h \rightarrow$ invisible $)<0.19(0.38)$ from global fits with the Higgs couplings to the SM fermions fixed to their SM values (varied freely while also allowing new particles in loops) [11] (see also [12-25]). This is a nontrivial constraint, since the SM Higgs decay width is so narrow. It essentially requires - with some caveats to be discussed below that the Higgs coupling to DM needs to be smaller than roughly the SM bottom Yukawa coupling, $y_{b} \sim \mathcal{O}(0.02)$. This then insures that the invisible branching ratio is smaller than the dominant channel, $h \rightarrow b \bar{b}$. 
On one hand we thus have a requirement that the Higgs should not couple too strongly to light DM. On the other hand, one needs $\mathcal{O}(1)$ couplings of Higgs to DM in order to obtain the correct thermal relic density. The tension between the two requirements leads to the apparent conclusion that the Higgs portal models with light DM are excluded. This was shown quantitatively in [26] for the simplest models by assuming that $\Gamma_{h}^{\text {invisible }} \lesssim 0.2 \Gamma_{h}^{\text {SM }} \simeq$ $0.8 \mathrm{MeV}$. Relaxing this bound by a factor of a few does not change the conclusion.

For heavier DM, $m_{\mathrm{DM}}>m_{h} / 2$, the bound on the invisible decay width of the Higgs is irrelevant. In this case one can search for DM using direct and indirect detection experiments. Existing constraints from direct DM detection are not stringent enough, but the next generation experiments are expected to cover most of the remaining viable parameter space $[27,28]$, with the exception of the parity violating Higgs portal where DM is a fermion [29]. This, on the other hand, can be covered in the future using indirect DM searches [29].

In this work we are primarily interested in the implications of an invisible Higgs decay signal (and the absence thereof so far) for light thermal relic DM. Are there still viable Higgs portal models with light DM? What modifications of the simplest models [26] are needed? The conclusion that the simplest versions of the Higgs portal are excluded by the bound on $\mathcal{B}(h \rightarrow$ invisible) utilizes effective field theory (EFT). The conclusion therefore relies on the assumption that an EFT description with the SM particles and DM as the only relevant dynamical degrees of freedom is valid both for the relic abundance calculation as well as for direct DM detection and Higgs phenomenology. For viable DM Higgs portals then either the EFT description (with naïve power counting) must be violated, or the invisible decay width of the Higgs is naturally suppressed. As we will show below this implies that given present experimental constraints, the Higgs can couple significantly to thermal relic DM with mass less than half of the Higgs only if there are other light particles in the theory (barring fine-tuned situations). In turn, should a nonzero invisible Higgs decay eventually be found and interpreted as a decay to thermal relic DM particles, then other new light particles need to be discovered.

To demonstrate this we first show in section 2 that extending the EFT description to higher dimensional operators but not enlarging the field content does not change the conclusions about the minimal DM Higgs portals if $h \rightarrow \mathrm{DM}+\mathrm{DM}$ decay is allowed. In section 3 we then show that for models where the two body Higgs decays to dark sector are forbidden, the scale of the EFT is small, $\Lambda \sim \mathcal{O}$ (few $100 \mathrm{GeV})$. This again implies that viable Higgs portals of DM require new light degrees of freedom beyond SM+DM. In section 4 we in turn give three examples of viable Higgs portal models of DM. Two models, described in subsections 4.1 and 4.2, can be matched onto EFT since the additional degrees of freedom are heavier - though not much heavier - than the Higgs. The two models do require fine-tuned cancellations in order to avoid experimental constraints. A model discussed in subsection 4.3, on the other hand, requires no such tunings. It contains, however, a particle lighter than DM and therefore violates the EFT assumptions. We summarize our conclusions in section 5. Details on direct DM detection, relic abundance calculations, and the fits to the Higgs data are relegated to the appendices A and B, respectively. 


\section{Higgs portals in effective field theory}

We start by reviewing the minimal Higgs portal scenarios. The SM is enlarged by a single neutral (DM) field, odd under a $Z_{2}$ symmetry. In the following we consider DM with spins up to and including spin 1, i.e. the possibility that DM is a scalar, $\phi$, a fermion, $\psi$ or a vector, $V_{\mu}$. The dominant interactions of DM with the SM are in each case, respectively,

$$
\begin{aligned}
\mathcal{H}_{\mathrm{eff}}^{0} & =\lambda^{\prime} H^{\dagger} H \times \phi^{\dagger} \phi, \\
\mathcal{H}_{\mathrm{eff}}^{1 / 2} & =\frac{c_{S}}{\Lambda} H^{\dagger} H \times \bar{\psi} \psi+\frac{i c_{P}}{\Lambda} H^{\dagger} H \times \bar{\psi} \gamma_{5} \psi, \\
\mathcal{H}_{\mathrm{eff}}^{1} & =\epsilon_{H} H^{\dagger} H \times V^{\mu} V_{\mu} .
\end{aligned}
$$

After electroweak (EW) symmetry breaking

$$
H^{\dagger} H \rightarrow \frac{1}{2}\left(v_{\mathrm{EW}}^{2}+2 v_{\mathrm{EW}} h+h^{2}\right),
$$

where $v_{\mathrm{EW}} \simeq 246 \mathrm{GeV}$ is the electroweak condensate and $h$ the Higgs boson. We see that the scalar and vector DM have renormalizable Higgs portal interactions with the SM. For fermion DM these interactions start only at dimension 5. In eq. (2.1b) $\Lambda$ is the scale at which the non-renormalizable DM-Higgs interactions are generated. In principle one can also write down higher dimensional operators that supplement (2.1a)-(2.1c), but are suppressed by more powers of $\Lambda$. The minimal Higgs portal models of DM assume $\Lambda \gg v_{\mathrm{EW}}, m_{\mathrm{DM}}$, such that the expansion $v_{\mathrm{EW}} / \Lambda$ makes sense and (2.1a)-(2.1c) are the dominant contributions to DM-SM interactions in the early universe and current experiments. As shown in [26], in all such models with light DM $\left(m_{\mathrm{DM}} \lesssim m_{h} / 2\right)$, the observed DM relic abundance is in conflict with the experimental bounds on the invisible decay width of the Higgs, while in the region $m_{\mathrm{DM}}>m_{h} / 2$, direct $\mathrm{DM}$ detection experiments are beginning to exclude the remaining parameter space.

But would the situation change if the $v_{\mathrm{EW}} / \Lambda$ expansion would not start at the lowest order, eqs. (2.1a)-(2.1c)? Can higher dimensional Higgs-DM operators [30] open new possibilities to reconcile Higgs portal DM with current experimental constraints? To answer this question we first perform a naïve dimensional analysis of the relevant processes based solely on the canonical dimension $(d=4+n)$ of the relevant interaction operator. For $m_{\mathrm{DM}} \ll m_{h} / 2$ the invisible Higgs branching fraction scales as

$$
\mathcal{B}(h \rightarrow \text { invisible }) \sim 10^{3}\left(\frac{m_{h}}{\Lambda}\right)^{2 n},
$$

where the overall normalization, $10^{3} \sim 1 / y_{b}^{2}$, is set by the total width of the SM Higgs. In (2.3) we used $v_{\mathrm{EW}} \sim m_{h}$, assumed that all dimensionless DM-Higgs couplings are $\mathcal{O}(1)$, and also assumed two-body $h \rightarrow$ invisible decay kinematics. In comparison, the current constraints from direct DM detection experiments give

$$
\frac{\left\langle\sigma_{\text {dir }}\right\rangle}{\left\langle\sigma_{\text {dir }}\right\rangle_{\text {excl. }}} \sim 10^{2}\left(\frac{m_{h}}{\Lambda}\right)^{2 n}\left(\frac{m_{\mathrm{DM}}}{m_{h}}\right)^{m} \beta^{2 m^{\prime}}
$$


where $m, m^{\prime}$ are non-negative integers, while the numerical pre-factor is simply the translation of the experimental limit due to XENON100 [31] and will increase in the future. Note that (2.4) assumes spin independent scattering since this is stronger than spin dependent one. The suppression in terms of $m_{h} / \Lambda$ is the same as for $\mathcal{B}(h \rightarrow$ invisible $)$, but depending on the operator structure there may be additional suppressions from typical DM velocity in the galactic halo, $\beta \sim 10^{-3}$, or from DM mass insertions, $m_{\mathrm{DM}} / m_{h}$. Both of these factors are smaller than one, therefore we conclude that at present for light DM the Higgs constraints are stronger than direct DM detection constraints for any operator dimension.

If DM is a thermal relic, then its abundance is fixed by thermal DM annihilation cross-section at the time of freeze-out,

$$
\left\langle\sigma_{\text {ann. }} v\right\rangle \propto \frac{y_{f}^{2}}{m_{h}^{2}}\left(\frac{m_{h}}{\Lambda}\right)^{2 n}\left(\frac{m_{\mathrm{DM}}}{m_{h}}\right)^{k},
$$

where $y_{f}$ is the SM Yukawa coupling for the heaviest open SM fermion channel, and $k \geqslant k_{\min }=0(2)$ for scalar and vector (fermion) DM with the equality sign for the lowest dimensional operators. In (2.5) we neglected relative velocity suppressions, $v_{r} \sim 0.4$, and as before set all Wilson coefficients to be $\mathcal{O}(1)$. In order to obtain the correct relic density, $\left\langle\sigma_{\text {ann. }} v\right\rangle \simeq 3 \cdot 10^{-26} \mathrm{~cm}^{3} / \mathrm{s}$, with $\Omega_{\mathrm{DM}} \propto 1 /\left\langle\sigma_{\text {ann. }} v\right\rangle$. From eq. (2.5) we then see that the correct relic density requires the scale $\Lambda$ to be lower if the dimensionality $n$ of the operator setting the annihilation cross section is higher. The scaling of $\mathcal{B} r(h \rightarrow$ invisible $)$ in terms of $\Lambda$ is the same as for $\left\langle\sigma_{\mathrm{ann}} v\right\rangle$, so that for the correct relic density one has

$$
\left(\frac{\mathcal{B}_{h}^{\text {invis. }}}{\left\langle\sigma_{\text {ann. }} v\right\rangle}\right)_{n} \sim\left(\frac{m_{h}}{m_{\mathrm{DM}}}\right)^{k-k_{\min }}\left(\frac{\mathcal{B}_{h}^{\text {invis. }}}{\left\langle\sigma_{\text {ann. }} v\right.}\right)_{n_{\min }},
$$

where $n_{\min }=4(5)$ for scalar and vector (fermion) DM. Since $k-k_{\min } \geqslant 0$, the Higgs constraints can only become stronger if the Higgs portal proceeds through higher dimensional operators. As a result, the higher dimensional operators cannot reconcile Higgs portal DM with the bounds on invisible Higgs branching ratio as long as $h \rightarrow \mathrm{DM}+\mathrm{DM}$ is possible and all couplings are $\mathcal{O}(1)$.

\section{Suppressed Higgs decays to dark sector}

In the previous section we saw that $\mathcal{B}(h \rightarrow$ invisible) places strong constraints on Higgs portals of DM. The analysis relied on two assumptions, i) that $h \rightarrow \mathrm{DM}+\mathrm{DM}$ decay is possible, and ii) that DM is the only light new physics particle. In this section we investigate in more details the first assumption, while the second assumption will be relaxed in the subsequent section. In the remainder of this section we therefore assume that $h \rightarrow \mathrm{DM}+\mathrm{DM}$ decay is forbidden either accidentally or due to the structure of the theory.

There are three possibilities to suppress the $h \rightarrow \mathrm{DM}+\mathrm{DM}$ decay. The first one is to assume DM annihilation to SM particles proceeds predominantly through operators not involving the Higgs. This possibility is orthogonal to the basic idea of a Higgs portal. It has also been studied extensively (cf. [32-36]) and we do not pursue it any further. The second possibility is that the $h \rightarrow \mathrm{DM}+\mathrm{DM}$ decay is kinematically forbidden simply because 
DM is heavy enough, $m_{\mathrm{DM}}>m_{h} / 2$. The final possibility is that DM couples through a special subset of Higgs portal operators, such that $h \rightarrow \mathrm{DM}+\mathrm{DM}$ decay is forbidden, while $h \rightarrow \mathrm{DM}+\mathrm{DM}+X_{\mathrm{SM}}$ is allowed, where $X_{\mathrm{SM}}$ denotes one or more SM particles in the final state. We set aside the model building question of how this is arranged in the UV theory and work within EFT. The $y_{b}^{2}$ suppression of the SM Higgs decay width is roughly of the same size as the phase space suppression from one or two additional final state particles. One may thus expect that $\mathcal{O}(1)$ couplings between DM and the Higgs would give at the same time the correct relic abundance as well as small enough $\mathcal{B}(h \rightarrow 2 \mathrm{DM}+X)$. Below we go through a list of possible operators, and as we will see a number of them are not excluded by direct and indirect DM detection constraints.

The simplest effective interactions generating $h \rightarrow \mathrm{DM}+\mathrm{DM}+X_{\mathrm{SM}}$ decays are built from the Higgs vector current

$$
H^{\dagger} \overleftrightarrow{D}^{\mu} H \equiv H^{\dagger} \overleftarrow{D}^{\mu} H-H^{\dagger} \vec{D}^{\mu} H \rightarrow \frac{i g}{2 c_{W}}\left(v_{\mathrm{EW}}^{2}+2 v_{\mathrm{EW}} h+h^{2}\right) Z^{\mu}
$$

where $c_{W}=\cos \theta_{W}$, with $\theta_{W}$ the weak mixing angle. The operators of the lowest dimension are [30]

$$
\begin{aligned}
\mathcal{H}_{\mathrm{eff}}^{0} & =\frac{c_{\phi}}{\Lambda^{2}} H^{\dagger} \overleftrightarrow{D}_{\mu} H \times \phi^{\dagger} \overleftrightarrow{\partial} \mu \phi \\
\mathcal{H}_{\mathrm{eff}}^{1 / 2} & =\frac{c_{\psi}^{V}}{\Lambda^{2}} i H^{\dagger} \overleftrightarrow{D}_{\mu} H \times \bar{\psi} \gamma^{\mu} \psi+\frac{c_{\psi}^{A}}{\Lambda^{2}} i H^{\dagger} \overleftrightarrow{D}_{\mu} H \times \bar{\psi} \gamma^{\mu} \gamma_{5} \psi \\
\mathcal{H}_{\mathrm{eff}}^{1} & =\frac{c_{V}}{\Lambda^{2}} i H^{\dagger} \overleftrightarrow{D}_{\nu} H \times V_{\mu} \overleftrightarrow{\partial^{\nu}} V^{\mu}
\end{aligned}
$$

For example, they appear in models where the DM is charged under a hidden $\mathrm{U}(1)$ gauge symmetry (spontaneously broken above the weak scale), exhibiting kinetic mixing with the SM hypercharge [37]. These operators induce a three body decay $h \rightarrow \mathrm{DM}+\mathrm{DM}+Z$, where for $Z \rightarrow \nu \bar{\nu}$ the decay would be completely invisible. They do not lead, however, to two body invisible decay $h \rightarrow \mathrm{DM}+\mathrm{DM}$. The three body Higgs decay is kinematically allowed if $m_{\mathrm{DM}}<\left(m_{h}-m_{Z}\right) / 2 \simeq 17 \mathrm{GeV}$. Such a light DM is subject to bounds from $Z \rightarrow E_{\text {miss }}$ measurements at LEP [39]. Requiring the correct relic density this constrains $m_{\mathrm{DM}}>24(34) \mathrm{GeV}$ for scalar (vector) DM, and $m_{\mathrm{DM}}>14(31) \mathrm{GeV}$ for fermionic DM with vector (axial-vector) interaction.

The operators in eq. (3.2) are also subject to severe direct DM detection constraints from $Z$-mediated DM scattering on nuclei (for details see appendix A). In figure 1 we show the predicted spin independent DM-nucleon cross sections (dashed blue lines) after requiring the correct thermal relic density $\Omega_{\mathrm{DM}} h^{2}=0.1186 \pm 0.0031$ [38]. The shaded blue regions indicate the validity of EFT, i.e., that $\Lambda \geq 2 m_{\mathrm{DM}}$. With the exception of fermionic DM with purely axial-vector interaction $\left(c_{\psi}^{V}=0\right)$ all parameter space allowed by relic density is excluded by XENON100 [31] (dot-dashed red lines). For fermionic DM with purely axial-vector interactions the spin-dependent cross section is plotted in figure 1, bottom right panel, since the SI cross-section is velocity suppressed. The result is compared to recent XENON100 bound on SD DM-neutron cross section [40], which excludes $m_{\mathrm{DM}}<35 \mathrm{GeV}$ and $50 \mathrm{GeV}<m_{\mathrm{DM}}<150 \mathrm{GeV}$. Note that the XENON1T [41] is 

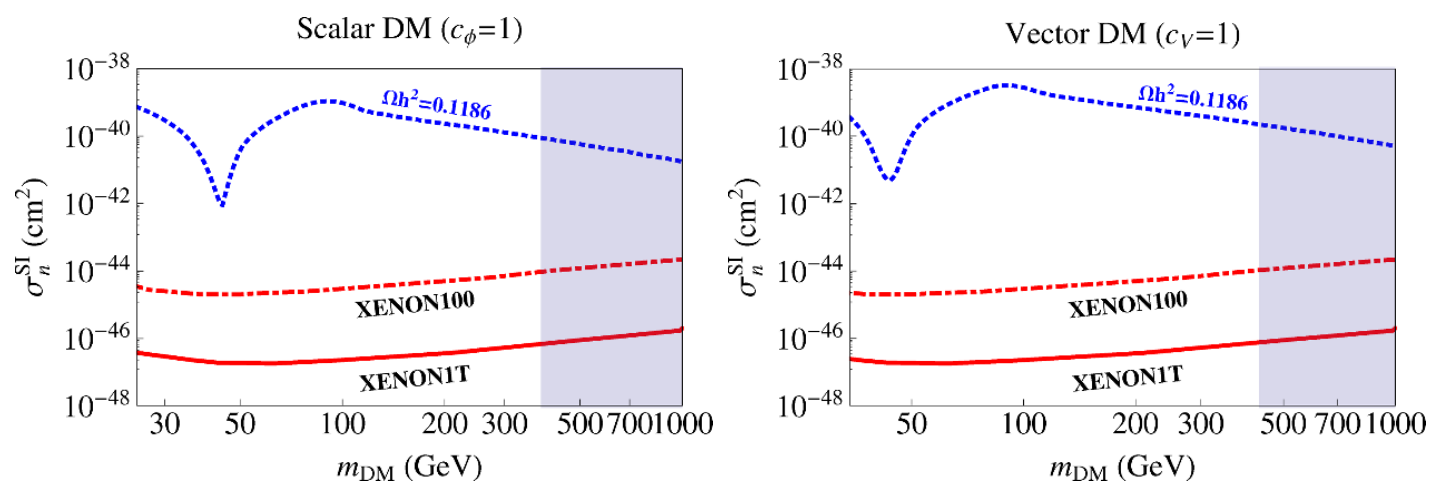

Fermionic DM $\left(c_{\psi}^{V}=1, c_{\psi}^{A}=0\right)$
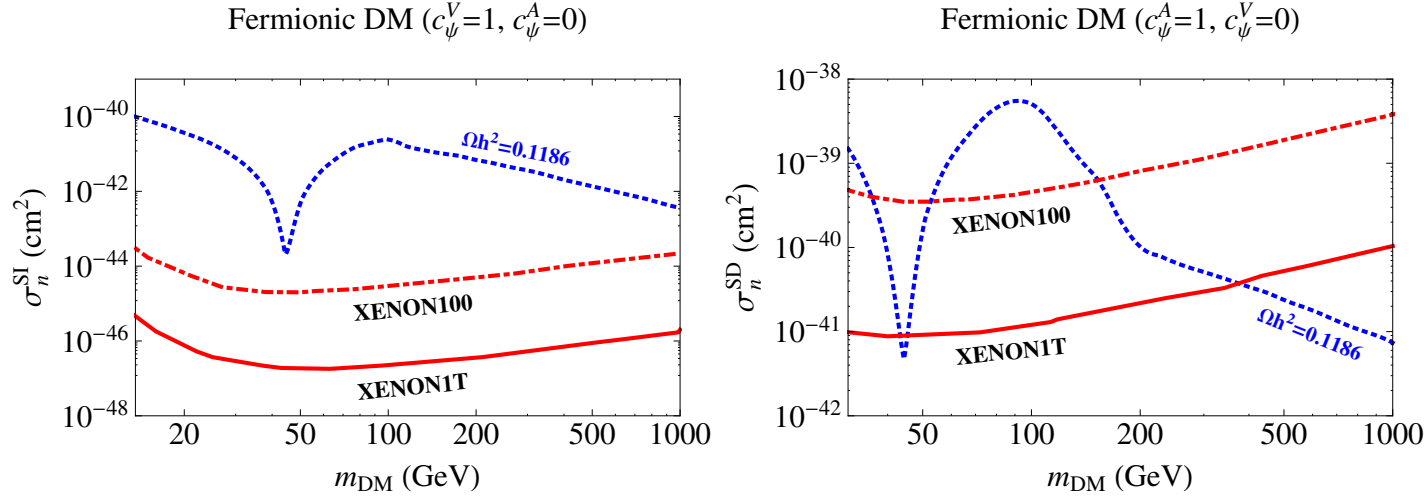

Figure 1. The spin independent DM-nucleon cross sections (dashed-blue) induced by Higgs vector current operators (3.2) after requiring correct thermal relic density $\Omega_{\mathrm{DM}} h^{2}=0.1186 \pm 0.0031$ [38] for scalar DM (top left), vector DM (top right) and fermion DM with vector (bottom left) couplings. Bottom right panel shows the spin dependent cross section for fermion DM with axial vector couplings. The current XENON100 [31] and projected future XENON1T bounds [27, 28] are denoted by dot-dashed and solid red lines, respectively. The shaded blue regions indicate where the EFT description breaks down $\left(\Lambda<2 m_{\mathrm{DM}}\right)$.

expected to cover almost completely the remaining low DM mass window. In summary, the combination of invisible $Z$ decay and direct DM detection constraints excludes any appreciable $\mathcal{B}(h \rightarrow$ invisible) from operators in eq. (3.2).

Another possibility is to couple DM to scalar or tensor fermionic currents. These automatically involve a Higgs field,

$$
\Gamma^{S}=H^{\dagger} \bar{D} Q, \quad H^{\dagger} \bar{E} L, \quad H^{* \dagger} \bar{U} Q, \quad \Gamma_{\mu \nu}^{T}=H^{\dagger} \bar{D} \sigma_{\mu \nu} Q, \quad H^{\dagger} \bar{E} \sigma_{\mu \nu} L, \quad H^{* \dagger} \bar{U} \sigma_{\mu \nu} Q .
$$

The lowest dimensional operators are then

$$
\begin{aligned}
\mathcal{H}_{\mathrm{eff}}^{0} & =\frac{f_{\phi}}{\Lambda^{2}} \Gamma^{S} \times \phi^{\dagger} \phi+\text { h.c. } \\
\mathcal{H}_{\mathrm{eff}}^{1 / 2} & =\frac{f_{\psi}^{S}}{\Lambda^{3}} \Gamma^{S} \times \bar{\psi} \psi+\frac{f_{\psi}^{P}}{\Lambda^{3}} \Gamma^{S} \times i \bar{\psi} \gamma_{5} \psi+\frac{f_{\psi}^{T}}{\Lambda^{3}} \Gamma_{\mu \nu}^{T} \times \bar{\psi} \sigma^{\mu \nu} \psi+h . c . \\
\mathcal{H}_{\mathrm{eff}}^{1} & =\frac{f_{V}}{\Lambda^{2}} \Gamma^{S} \times V_{\mu} V^{\mu}+\text { h.c. }
\end{aligned}
$$

where the dependence of couplings on SM fermion flavors is implicit. Operators involving $\Gamma^{S}$ can be generated for example in models with extended scalar sectors, as we will discuss 
below. On the other hand, the generation of tensorial $\Gamma_{\mu \nu}^{T}$ interactions is typically more involved. One possibility is to introduce a SM-DM mediator sector with a gauge symmetry under which both SM and DM are neutral. The appropriate irrelevant couplings to generate the tensorial SM-DM interaction can then possibly be obtained at the loop level. A complete model construction is thus quite intricate and beyond our scope, so we do not pursue it any further.

We first assume the couplings in eqs. (3.4) to be proportional to the fermion masses,

$$
f_{\phi}=\frac{\sqrt{2} m_{f}}{v_{E W}}, \quad f_{\psi}^{S, P, T}=\frac{\sqrt{2} m_{f}}{v_{E W}}, \quad f_{V}=\frac{\sqrt{2} m_{f}}{v_{E W}},
$$

so that possible flavor changing neutral currents (FCNCs) are automatically suppressed. The operators in eq. (3.4) lead to four body Higgs decays, that are unobservably small. For instance, assuming thermal relic DM with $m_{\mathrm{DM}}=20 \mathrm{GeV}$ one has $\mathcal{B}(h \rightarrow \mathrm{DM}+\mathrm{DM}+b \bar{b}) \sim$ $\mathcal{O}\left(10^{-7}\right)$ for both purely pseudo scalar and purely tensorial DM interactions.

Figure 2 shows the predictions for the spin-independent DM-nucleon cross sections in the upper four panels, for scalar DM, vector DM, and fermion DM with scalar and pseudoscalar interactions, respectively (blue dashed lines), requiring correct thermal relic DM abundance. The spin-dependent cross section for fermion DM with purely tensorial interaction is shown in the lower panel in figure 2. For the chosen flavor structure of the relevant couplings, eq. (3.5), XENON100 bounds (dot-dashed red lines) exclude almost all possibilities except for fermionic DM with parity-violating or tensorial interactions. The parity violating fermionic DM evades the current XENON100 and also the projected XENON1T bound (red solid line) because the scattering cross section is velocity suppressed. The direct detection cross section for the tensorial interactions is strongly suppressed by the assumption that the coupling to light quarks is suppressed by light quark masses, eq. (3.5) (unlike for scalar interactions this suppression carries over for tensor interactions when matching from quark to nucleon level operators, see refs. [42] and [43] for further details).

The remaining two possibilities are constrained by indirect DM searches. In figure 3 we compare the bounds on annihilation cross sections $\langle\sigma v\rangle$ for $b \bar{b}$ (blue lines) and $\tau^{+} \tau^{-}$ (red lines) channels $[44,45]$ with the predictions from the last two operators in eq. (3.4), when correct relic density is assumed in the predictions. We see that the fermionic DM with pseudo-scalar or tensorial interactions is constrained to be heavier than $m_{\mathrm{DM}}>15 \mathrm{GeV}$. For reference we also show in figure 3 the possibility of Higgs portal coupling to DM through the axial-vector operator from eq. (3.2), which is not excluded by direct detection. It demonstrates that for $Z$ mediated channels, the constraints from indirect detection are not as significant. The reason lies in the assumed flavor structure. This is fixed for operators in eq. (3.2) by the couplings of the $Z$. DM then annihilates to all fermions democratically, reducing the signal in the $b \bar{b}$ and $\tau^{+} \tau^{-}$final states. For the flavor structure assumed in eq. (3.4) these are the dominant channels, however, making the constraints more powerful.

This also highlights the fact that the bounds on operators in eq. (3.4) depend strongly on the assumed flavor structure of the Wilson coefficients. We do not attempt to cover all possibilities but rather only entertain a few representative cases. For instance, increasing the couplings to light quarks, $u, d, s$, the direct DM detection bounds would become sig- 
Scalar DM

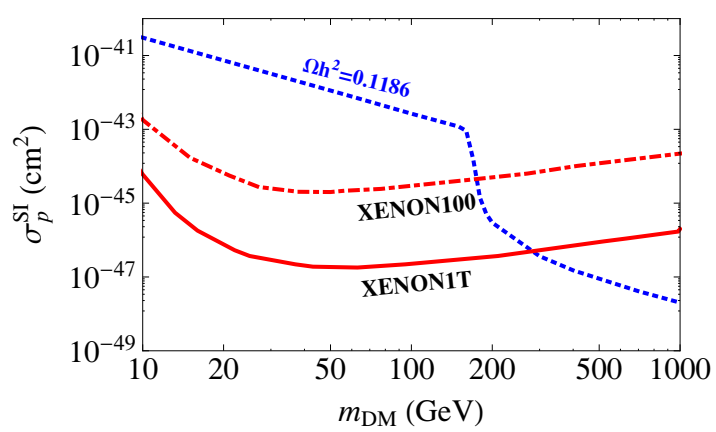

Fermionic DM $\left(f_{\psi}^{S}=\sqrt{2} m_{f} / v_{\mathrm{EW}}, f_{\psi}^{P, T}=0\right)$

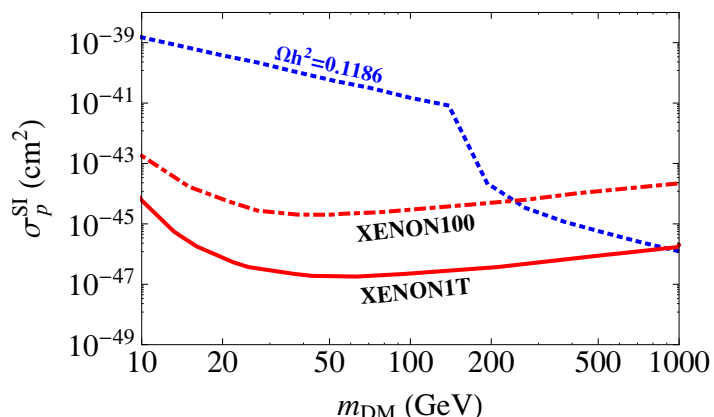

Vector DM

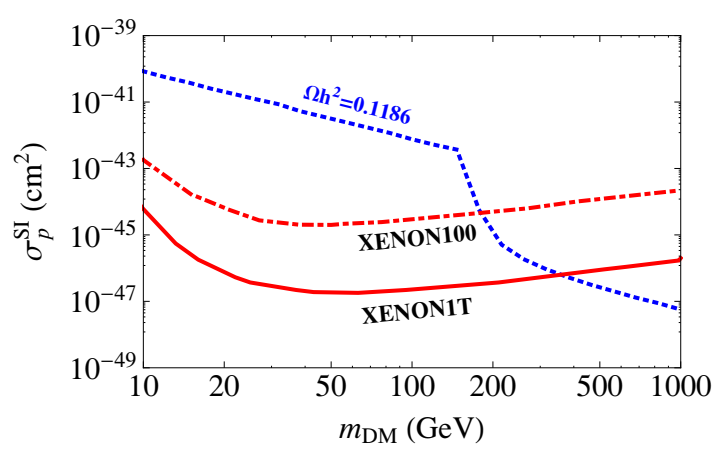

Fermionic DM $\left(f_{\psi}^{P}=\sqrt{2} m_{f} / v_{\mathrm{EW}}, f_{\psi}^{S, T}=0\right)$

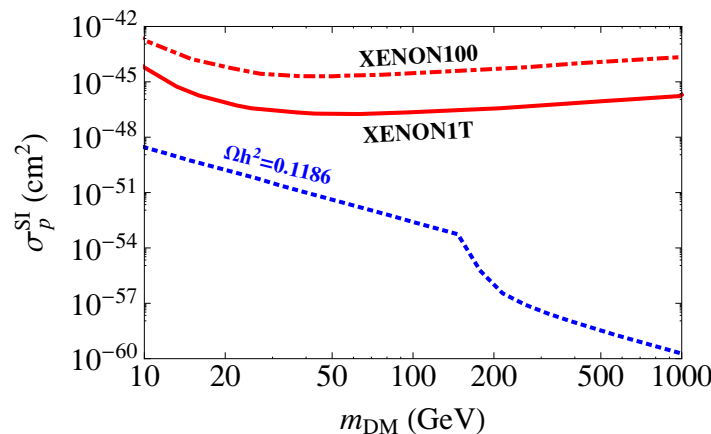

Fermionic DM $\left(f_{\psi}^{T}=\sqrt{2} m_{f} / v_{\mathrm{EW}}, f_{\psi}^{S, P}=0\right)$

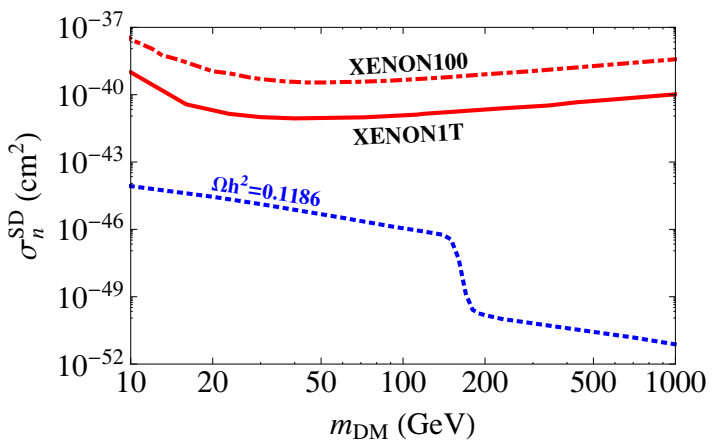

Figure 2. The DM-nucleon cross sections (dashed-blue) induced by operators (3.4). The predicted values are compared to the current XENON100 bound (dot-dashed-red line) and future XENON1T bound (continuous-red line).

nificantly stronger, while the relic density would remain practically unaffected. Note that in the limit where DM does not couple to the light quarks but only to $3^{\text {rd }}$ generation, the direct detection bounds are still relevant since one induces interactions to gluons at loop level. An interesting possibility is to have Wilson coefficients differ in sign such that the DM-nucleon elastic scattering cross-section vanishes. This possibility was pointed out in the context of type II Two-Higgs-Doublet Model (2HDM-II) in ref. [46], to be discussed in more detail in subsection 4.2. Another possibility where direct detection bounds are weak or completely irrelevant is the case of leptophilic DM, where the Wilson coefficients for operators coupling to quarks in eq. (3.4) are suppressed [47]. 

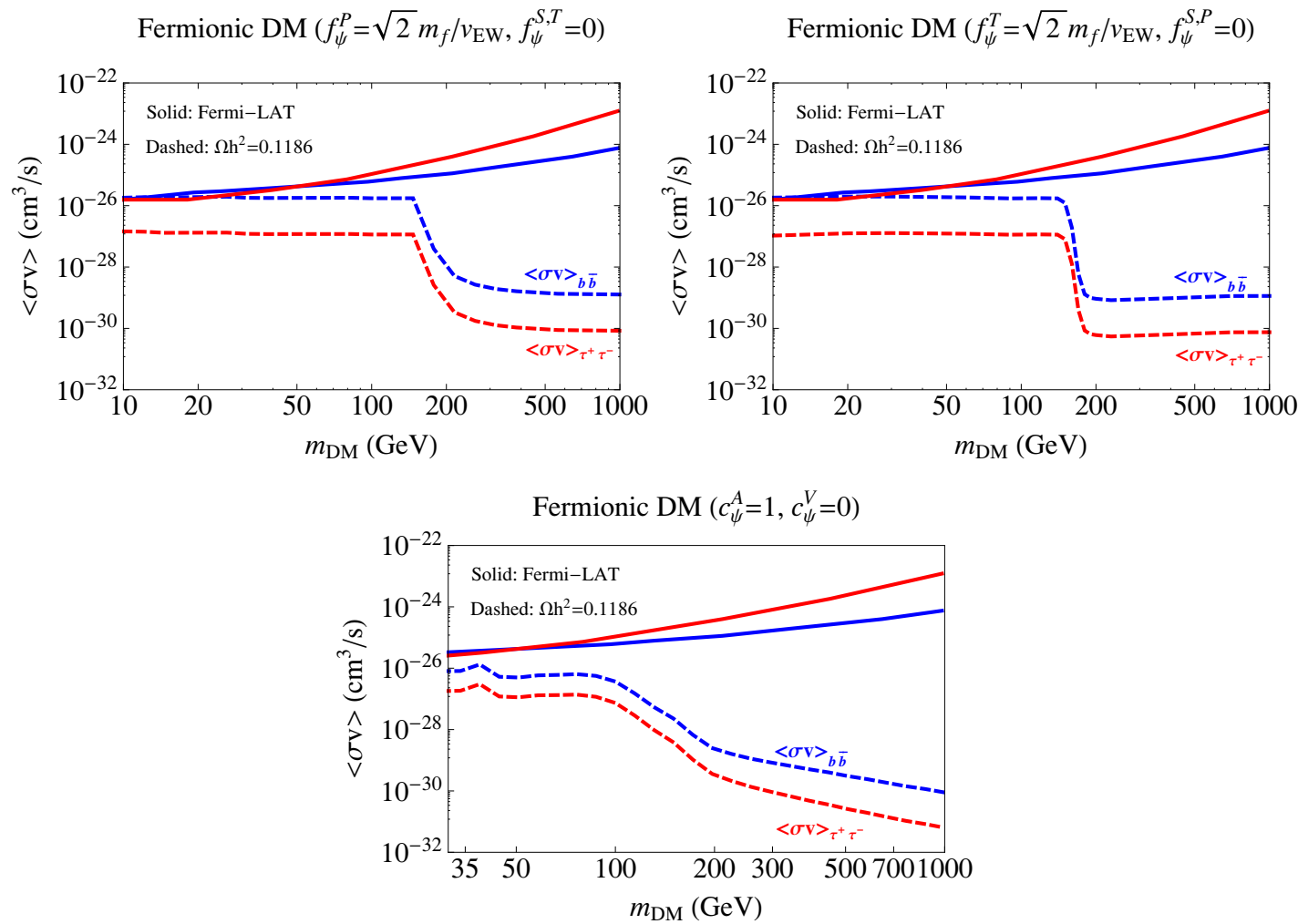

Figure 3. The $b \bar{b}$ (blue) and $\tau^{+} \tau^{-}$(red) annihilation cross-sections $(\langle\sigma v\rangle)$ for the fermionic operators in (3.4) (upper two panels) and for fermionic DM with axial vector coupling to Higgs vector current in (3.2) $\left(c_{\psi}^{V}=0\right)$. The continuous (dashed) lines indicate the present experimental upper bounds $[44,45]$ (predicted values assuming correct DM relic density) on $\langle\sigma v\rangle$.

Regardless of the detailed flavor structure all these operators do have one feature in common. To obtain correct relic abundance the EFT cut-off scale $\Lambda$ is required to be low, $\mathcal{O}$ (few $100 \mathrm{GeV}$ ). The important parameters here are the values of Wilson coefficients $f_{\phi}, f_{\psi}^{S, P, T}, f_{V}$ for bottom quarks in the currents (3.3) and the value of the Higgs bottom Yukawa coupling (or if this is suppressed, the largest Yukawa coupling among the open annihilation channels). From Higgs data we know that the Higgs bottom Yukawa cannot be significantly larger than the SM value. Using the SM value for $y_{b}$ we show in figure 4 the dependence of $\Lambda$ on $m_{\mathrm{DM}}$ for scalar and tensor fermionic operators (3.4), setting $f_{\psi}^{S}=$ $f_{\psi}^{T}=y_{b}$ as in eq. (3.5). Since the annihilation cross section scales as $f_{\psi}^{2} / \Lambda^{6}$ for fermionic DM, taking $f_{\psi} \sim \mathcal{O}(1)$ still leads to $\Lambda \lesssim 600 \mathrm{GeV}$ for $m_{\mathrm{DM}}<m_{h} / 2$. This means that in any case a viable Higgs portal of light DM using operators in eq. (3.4) will require new particles with weak scale masses beside DM itself.

Finally, DM can couple to the Higgs through Weinberg-like operator,

$$
L^{i} L^{j} H^{k} H^{l} \epsilon_{i k} \epsilon_{j l} \times \mathcal{O}_{\text {dark }},
$$

where $i, j, k, l$ are $\mathrm{SU}(2)_{L}$ indices, $\epsilon_{i j}$ is the antisymmetric tensor with $\epsilon_{12}=-\epsilon_{21}=1$, and 


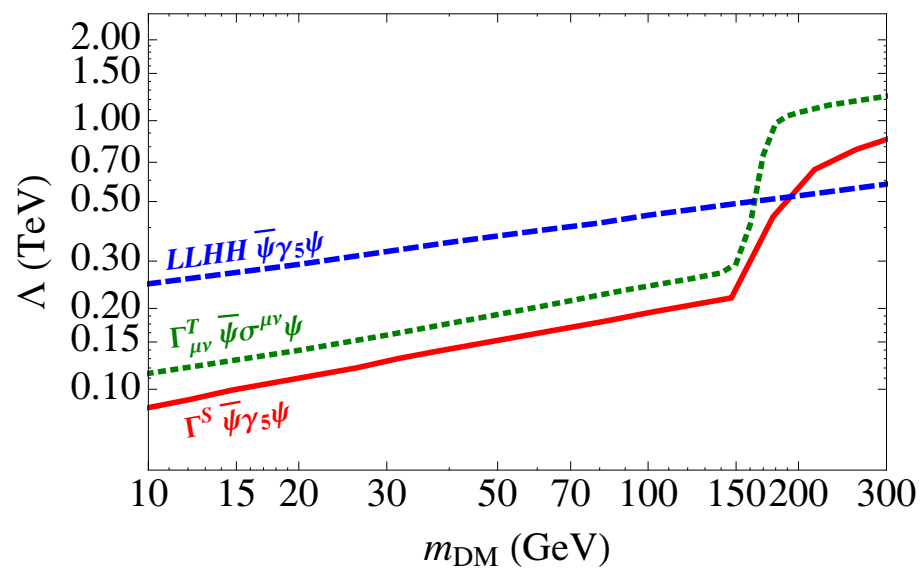

Figure 4. The dependence of the EFT cut-off scale $\Lambda$ for scalar (blued dashed line) and tensor (green dotted line) operators (3.4) and flavor structure (3.5) for fermionic DM as a function of DM mass $m_{\mathrm{DM}}$ after requiring correct relic density. The solid red line shows $\Lambda$ for leptonic operator (3.7).

$\mathcal{O}_{\text {dark }}$ the DM operator. The lowest dimensional interactions are explicitly,

$$
\begin{aligned}
\mathcal{H}_{\mathrm{eff}}^{0} & =\frac{g_{\phi}}{\Lambda^{3}} L^{i} L^{j} H^{k} H^{l} \epsilon_{i k} \epsilon_{j l} \times \phi^{\dagger} \phi \\
\mathcal{H}_{\mathrm{eff}}^{1 / 2} & =\frac{g_{\psi}^{S}}{\Lambda^{4}} L^{i} L^{j} H^{k} H^{l} \epsilon_{i k} \epsilon_{j l} \times \bar{\psi} \psi+\frac{g_{\psi}^{P}}{\Lambda^{4}} L^{i} L^{j} H^{k} H^{l} \epsilon_{i k} \epsilon_{j l} \times i \bar{\psi} \gamma_{5} \psi, \\
\mathcal{H}_{\mathrm{eff}}^{1} & =\frac{g_{V}}{\Lambda^{3}} L^{i} L^{j} H^{k} H^{l} \epsilon_{i k} \epsilon_{j l} \times V_{\mu} V^{\mu},
\end{aligned}
$$

and similar operators with $\phi^{\dagger} \phi \rightarrow \phi \phi, \bar{\psi} \psi \rightarrow \bar{\psi}^{C} \psi$ and $\bar{\psi} \gamma_{5} \psi \rightarrow \bar{\psi}^{C} \gamma_{5} \psi$ replacements. The operators in eqs. (3.7) contribute to neutrino masses at one loop. Modulo cancellations, this suppresses all the operators well below the level required for the thermal scattering cross-section to give the observed DM relic density. The only exception is the fermionic DM operator with purely pseudo-scalar interaction $\left(g_{\psi}^{P}\right)$ whose loop contributions to neutrino masses vanish identically by parity invariance, and the $\phi \phi, \bar{\psi}^{C} \psi, \bar{\psi}^{C} \gamma_{5} \psi$ type operators if DM carries (conserved) lepton number. The resulting invisible Higgs decay governed by the $g_{\psi}^{P}$ interaction is very suppressed, that is, $\mathcal{B}(h \rightarrow \mathrm{DM}+\mathrm{DM}+\bar{\nu} \bar{\nu}) \simeq 10^{-7}$ for $m_{\mathrm{DM}}=20 \mathrm{GeV}$ and assuming correct relic DM abundance. Note that the operator $L^{i} L^{j} H^{k} H^{l} \epsilon_{i k} \epsilon_{j l} \times i \bar{\psi} \gamma_{5} \psi$ does induce DM-nucleon scattering, but only at loop level and the contribution is furthermore proportional to neutrino mass. The DM-nucleon cross section, therefore, is very suppressed.

The DM annihilation cross section induced by the $L^{i} L^{j} H^{k} H^{l} \epsilon_{i k} \epsilon_{j l} \times i \bar{\psi} \gamma_{5} \psi$ operator is given by

$$
\sigma_{\psi \bar{\psi} \rightarrow \bar{\nu} \bar{\nu}}=\frac{v_{E W}^{4}\left(g_{\psi}^{P}\right)^{2}}{64 \pi \Lambda^{8}} \frac{s}{\sqrt{1-\beta\left(m_{\mathrm{DM}}^{2}\right)}},
$$


with $\beta\left(M^{2}\right) \equiv 4 M^{2} / s$ and $s \simeq 4 m_{\mathrm{DM}}^{2}$ is the energy in the center of mass frame. The value of $\Lambda$ required to obtain the correct relic density is shown in figure 4 (red solid line), assuming only one neutrino flavor in the final state and setting $g_{\psi}^{p}=1$. We observe that the required scale is again low, i.e. for $m_{\mathrm{DM}}=40 \mathrm{GeV}, \Lambda \simeq 300 \mathrm{GeV}$.

In conclusion, our discussion in this section shows that even if the invisible branching ratio of the Higgs is suppressed, viable Higgs portals to light thermal relic DM require new particles with masses of a few $100 \mathrm{GeV}$.

\section{Examples of viable Higgs portal models}

One of the main results of the previous two sections is that Higgs portal models of light DM are still viable, however SM cannot be extended just by DM. Extra light particles are required. The main new ingredient is that the presence of extra light particles increases the DM annihilation cross section, so that correct relic abundance is obtained. Below we show three examples of viable Higgs portal models of light DM. The first two examples illustrate models that match onto EFT discussion of the previous section. In the first example we add to SM and DM an extra electroweak triplet and a singlet (subsection 4.1). This is a realization of a leptophilic model that generates an operator in eq. (3.7). The second example is a Two Higgs Doublet Model of type II with an addition of a scalar DM field (subsection 4.2). It generates EFT operators in eq. (3.4). The third example violates EFT assumptions since we add to SM and DM an extra scalar singlet that is lighter than DM (subsection 4.3). As we will see, the value of $\mathcal{B}(h \rightarrow$ invisible) is model dependent. It can be $\mathcal{O}(1)$ as in our example in subsection 4.3 , or can be suppressed by the assumed structure of the theory as in the two examples in subsections 4.1 and 4.2 .

\subsection{SM + DM with an extra triplet and a singlet}

In this section, we present a model that could generate the operator $L^{i} L^{j} H^{k} H^{l} \epsilon_{i k} \epsilon_{j l} \times$ $i \bar{\psi} \gamma_{5} \psi$. As we will see shortly, it can be done by extending SM particle content by a Dirac fermion DM $(\psi)$, an electroweak singlet scalar $(\phi)$, and an electroweak triplet scalar $(\Delta)$. The extra fields therefore transform under the $\mathrm{SM}$ gauge group $\mathrm{SU}(3)_{C} \times \mathrm{SU}(2)_{L} \times \mathrm{U}(1)_{Y}$ as

$$
\psi \sim(1,1,0), \quad \phi \sim(1,1,0), \quad \Delta \sim(1,3,1) .
$$

We use the notation in which $\Delta$ is represented by the $2 \times 2$ matrix,

$$
\Delta=\left(\begin{array}{cc}
\Delta^{+} / \sqrt{2} & \Delta^{++} \\
\Delta^{0} & -\Delta^{+} / \sqrt{2}
\end{array}\right) .
$$

We introduce the following interactions

$\mathcal{L} \supset-\frac{m_{\phi}^{2}}{2} \phi^{2}-m_{\Delta}^{2} \operatorname{Tr} \Delta^{\dagger} \Delta-m_{\mathrm{DM}} \bar{\psi} \psi+\left[i y \bar{\psi} \gamma_{5} \psi \phi+\lambda \phi H^{i} H^{j} \epsilon_{i k} \Delta_{j k}^{*}+f_{a b} L_{a}^{i} L_{b}^{j} \epsilon_{i k} \Delta_{k j}+\right.$ h.c. $]$,

where $H$ is the usual SM Higgs doublet, $a, b=1,2,3$ are generation indices, $i, j, k$ are $\mathrm{SU}(2)_{L}$ indices, and $\epsilon_{i j}$ is the antisymmetric tensor. In the above Lagrangian, the $\phi$ is 
assumed to be a real scalar. Note that we have written only terms relevant to generate the $L^{i} L^{j} H^{k} H^{l} \epsilon_{i k} \epsilon_{j l} \times i \bar{\psi} \gamma_{5} \psi$ operator, which is obtained after integrating out $\phi$ and $\Delta$.

It is worth mentioning that one could also consider a variation of the above model in which lepton number is preserved. In this case, the dark matter fermion carries a lepton number -1 and the Lagrangian is modified to

$\mathcal{L} \supset-m_{\phi}^{2} \phi^{*} \phi-m_{\Delta}^{2} \operatorname{Tr} \Delta^{\dagger} \Delta-m_{\mathrm{DM}} \bar{\psi} \psi+\left[y \bar{\psi}^{C} \psi \phi+\lambda \phi H^{i} H^{j} \epsilon_{i k} \Delta_{j k}^{*}+f_{a b} L_{a}^{i} L_{b}^{j} \epsilon_{i k} \Delta_{k j}+\right.$ h.c. $]$

with $\phi$ complex in this case.

From now on, we shall focus on the model given in eq. (4.3). The Lagrangian (4.3) could be supplemented by several other gauge-invariant terms such as

$$
H^{T} \Delta^{\dagger} H, \quad \phi \operatorname{Tr} \Delta^{\dagger} \Delta, \quad H^{\dagger} H \phi, \quad H^{\dagger} H \operatorname{Tr} \Delta^{\dagger} \Delta, \quad \operatorname{Tr}\left(\Delta^{\dagger} \Delta\right)^{2}, \quad\left(\operatorname{Tr} \Delta^{\dagger} \Delta\right)^{2}, \quad H^{\dagger} \Delta^{\dagger} \Delta H .
$$

Some of them are already phenomenologically constrained to be small. For instance, $H^{T} \Delta^{\dagger} H$ would generate neutrino masses once $\Delta$ is integrated out [48-50]. Its coefficient therefore must be very small, much smaller than $m_{\Delta}$.

By the same reasoning, the term $\mu H^{\dagger} H \phi$ should be suppressed too. The simultaneous presences of $f_{a b} L_{a} L_{b} \Delta, \lambda \phi H^{T} H \Delta^{\dagger}$, and $\mu H^{\dagger} H \phi$ terms breaks lepton number by two units, and as a result the neutrino masses are generated at tree level. To generate unsuppressed Weinberg-like operator (3.7) we require $f \sim \lambda \sim 1$ and $m_{\phi} \sim$ few hundreds GeV, so that $\mu$ needs to be very small, i.e., $\mu \lesssim 1 \mathrm{eV}$. Consequently, the $\phi-h$ mixing is extremely suppressed and cannot induce sizeable $h \rightarrow \mathrm{DM}+\mathrm{DM}$ decay nor DM-nucleon elastic cross section. The invisible Higgs decay can thus only occur through the 4-body mode $h \rightarrow$ $\bar{\nu} \bar{\nu}+\mathrm{DM}+\mathrm{DM}$ with branching ratio of $\sim 10^{-6}$ for $m_{D M}=40 \mathrm{GeV}$. This number is much too small to be measured in the near future.

The correct DM relic density is obtained from $\bar{\psi} \psi \rightarrow \bar{\nu} \bar{\nu}$ annihilation that can proceed through $s$-channel $\phi$ and $\Delta^{0}$ virtual states. The annihilation is unsuppressed as long as there is significant mixing between $\phi$ and $\Delta^{0}$ states through the $\lambda \phi H^{i} H^{j} \epsilon_{i k} \Delta_{j k}^{*}$ term (after electroweak symmetry breaking). In figure 5 we show as a function of $m_{\mathrm{DM}}$ the required $m_{\Delta}$ and the masses $m_{1,2}$ of the two $\phi-\Delta^{0}$ mixed physical states such that the observed $\mathrm{DM}$ relic density is generated. The numerical example shown is for maximal mixing, where $m_{\phi}=m_{\Delta}$, and we set $f_{a b}=y=\lambda=1$. As anticipated, the required extra states are light, with masses of the order of the weak scale.

The fact that viable Higgs portal models with light DM require additional light states can have phenomenological implications beyond dark matter searches. In the present model, for instance, there are two charged scalars, $\Delta^{++}$and $\Delta^{+}$. These can mediate lepton flavor violating (LFV) processes such as $\ell_{a} \rightarrow \ell_{b} \gamma$ and $\ell_{a}^{-} \rightarrow \ell_{b}^{+} \ell_{c}^{-} \ell_{d}^{-}$. The radiative decays can arise at one-loop mediated by either $\Delta^{+}$or $\Delta^{++}$particles, with the rate

$$
\Gamma\left(\ell_{a} \rightarrow \ell_{b} \gamma\right)=\frac{m_{\ell_{a}}^{5} \alpha_{e m}}{\left(24 \pi^{2}\right)^{2}}\left(f^{\dagger} f\right)_{a b}^{2}\left(\frac{1}{8 m_{\Delta^{+}}^{2}}+\frac{1}{m_{\Delta^{++}}^{2}}\right)^{2}
$$




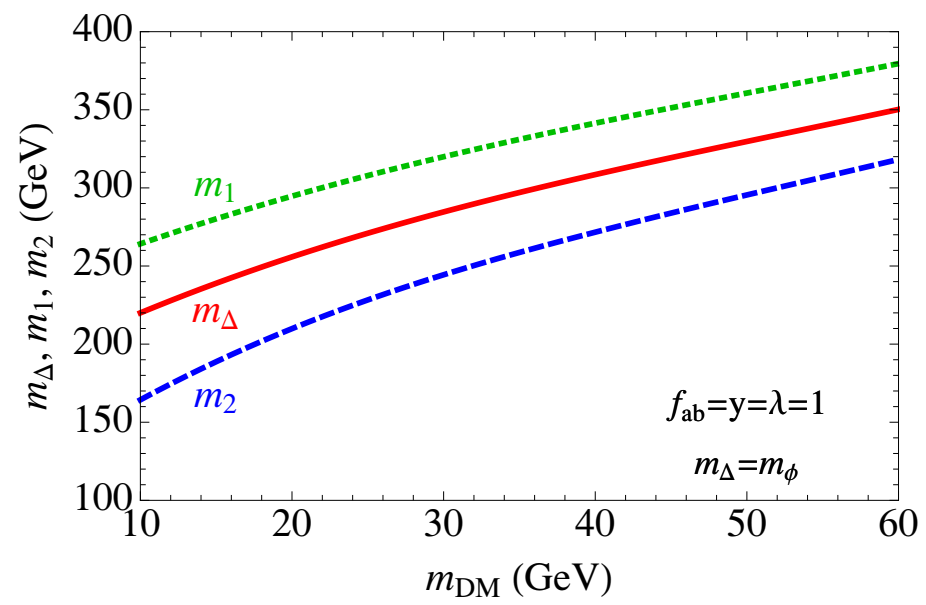

Figure 5. The dependence on $m_{\mathrm{DM}}$ of the parameter $m_{\Delta}$ (red solid line) in the SM + DM model with an extra triplet and a singlet Lagrangian (4.3) for which proper relic density is obtained. The masses of physical $\phi-\Delta^{0}$ mixed states, $m_{1,2}$ are shown as blue dashed and green dotted lines. Other inputs in (4.3) are set to $f_{a b}=y=\lambda=1$ with $m_{\phi}=m_{\Delta}$.

where $\alpha_{e m}$ is the QED fine-structure constant. The $\ell_{a}^{-} \rightarrow \ell_{b}^{+} \ell_{c}^{-} \ell_{d}^{-}$decay can proceed through tree-level $\Delta^{++}$exchange, giving

$$
\Gamma\left(\ell_{a}^{-} \rightarrow \ell_{b}^{+} \ell_{c}^{-} \ell_{d}^{-}\right)=\frac{1}{2\left(1+\delta_{c d}\right)} \frac{m_{\ell_{a}}^{5}}{192 \pi^{3}}\left|\frac{f_{a b} f_{c d}}{m_{\Delta^{++}}^{2}}\right|^{2},
$$

where $\delta_{c d}$ encodes the symmetry factor for two identical particles in the final state [51]. The resulting bounds on $f_{a b}$ from various LFV processes are given in table 1 for the case of $m_{\Delta^{+}}=m_{\Delta^{++}}=m_{\Delta}$. (For previous study of LFV in the triplet model, see refs. [53-55].) For $m_{\Delta}=220-350 \mathrm{GeV}$ as required by the relic abundance, the off-diagonal $f_{a b}$ are severely constrained. There are also bounds on diagonal couplings from collider searches. For flavor degenerate case, with $f_{a a}=1$ for $a=1,2,3$, the CMS Collaboration [56] reports a bound $m_{\Delta}>403 \mathrm{GeV}$, which is inconsistent with the relic DM density requirement. The search is less effective for $f_{\tau \tau}=1$ and $f_{e e}=f_{\mu \mu}=0$, in which case $\Delta^{--}$decays exclusively into same-sign tau pairs. The lower limit on $\Delta^{++}$mass is then $m_{\Delta}>204 \mathrm{GeV}$ [56], so that correct relic density can still be obtained.

\subsection{HDM-II + DM}

Our next example of a viable Higgs portal DM is a type II Two-Higgs-Doublet-Model (2HDM-II) supplemented by an extra singlet scalar - the DM. This is the simplest realization of the fermionic operators in eq. (3.4), discussed in the previous section assuming EFT. While phenomenologically viable, the model does have two ad-hoc features. The invisible Higgs decay width is suppressed by dialling down the appropriate dimensionless parameter, while direct DM detection bounds are avoided by fine-tuning the parameters so that two competing operator contributions cancel to a large extent. 


\begin{tabular}{|lcc|}
\hline Process & Branching ratio bound & Bounds on $f_{a b}$ \\
\hline$\mu^{-} \rightarrow e^{+} e^{-} e^{-}$ & $1.0 \times 10^{-12}$ & $\left|f_{e e} f_{e \mu}\right|<2.8 \times 10^{-5}\left(m_{\Delta} / \mathrm{TeV}\right)^{2}$ \\
$\tau^{-} \rightarrow e^{+} e^{-} e^{-}$ & $2.7 \times 10^{-8}$ & $\left|f_{e e} f_{e \tau}\right|<0.01\left(m_{\Delta} / \mathrm{TeV}\right)^{2}$ \\
$\tau^{-} \rightarrow e^{+} e^{-} \mu^{-}$ & $1.8 \times 10^{-8}$ & $\left|f_{e \mu} f_{e \tau}\right|<0.007\left(m_{\Delta} / \mathrm{TeV}\right)^{2}$ \\
$\tau^{-} \rightarrow e^{+} \mu^{-} \mu^{-}$ & $1.7 \times 10^{-8}$ & $\left|f_{\mu \mu} f_{e \tau}\right|<0.009\left(m_{\Delta} / \mathrm{TeV}\right)^{2}$ \\
$\tau^{-} \rightarrow \mu^{+} e^{-} e^{-}$ & $1.5 \times 10^{-8}$ & $\left|f_{e e} f_{\mu \tau}\right|<0.008\left(m_{\Delta} / \mathrm{TeV}\right)^{2}$ \\
$\tau^{-} \rightarrow \mu^{+} \mu^{-} e^{-}$ & $2.7 \times 10^{-8}$ & $\left|f_{e \mu} f_{\mu \tau}\right|<0.009\left(m_{\Delta} / \mathrm{TeV}\right)^{2}$ \\
$\tau^{-} \rightarrow \mu^{+} \mu^{-} \mu^{-}$ & $2.1 \times 10^{-8}$ & $\left|f_{\mu \mu} f_{\mu \tau}\right|<0.01\left(m_{\Delta} / \mathrm{TeV}\right)^{2}$ \\
$\mu \rightarrow e \gamma$ & $5.7 \times 10^{-13}$ & $\left|f_{\mu a}^{*} f_{a e}\right|<2.7 \times 10^{-4}\left(m_{\Delta} / \mathrm{TeV}\right)^{2}$ \\
$\tau \rightarrow e \gamma$ & $3.3 \times 10^{-8}$ & $\left|f_{\tau a}^{*} f_{a e}\right|<0.15\left(m_{\Delta} / \mathrm{TeV}\right)^{2}$ \\
$\tau \rightarrow \mu \gamma$ & $4.4 \times 10^{-8}$ & $\left|f_{\tau a}^{*} f_{a \mu}\right|<0.18\left(m_{\Delta} / \mathrm{TeV}\right)^{2}$ \\
\hline
\end{tabular}

Table 1. The bounds on LFV couplings $f_{a b}$ of $\Delta$ in eq. (4.3), following from leptonic LFV decays. The experimental 95\% C.L. upper bounds are from [39], except for $\mu \rightarrow e \gamma$ which is from [52]. We set $m_{\Delta^{+}}=m_{\Delta^{++}}=m_{\Delta^{\prime}}$.

The detailed structure of the model is as follows. The particle content consists of SM fermions, two Higgs doublets, $H_{1}$ and $H_{2}$, and an extra real scalar $S$. Under SM gauge group, these scalars transform as

$$
H_{1} \sim(1,2,1 / 2), \quad H_{2} \sim(1,2,1 / 2), \quad S \sim(1,1,0) .
$$

The singlet $S$ is assumed to be $Z_{2}$ odd and is identified as DM. The Yukawa interactions of the two doublets are assumed to be the same as in type II $2 \mathrm{HDM} ; H_{1}$ couples to $d_{R}$ and $e_{R}$, while $H_{2}$ only couples to $u_{R}$,

$$
\mathcal{L}_{Y}=-Y_{u} \bar{Q} \tilde{H}_{2} u_{R}-Y_{d} \bar{Q} H_{1} d_{R}-Y_{\ell} \bar{L} H_{1} e_{R}+\text { h.c. }
$$

where $\tilde{H}_{i} \equiv i \sigma_{2} H_{i}^{*}$ and $H_{i}=\left(H_{i}^{+},\left(v_{i}+h_{i}+i \chi_{i}\right) / \sqrt{2}\right)$. DM couples directly to the two Higgs doublets,

$$
\mathcal{L} \supset \frac{\lambda_{S 1}}{2} S^{2}\left(H_{1}^{\dagger} H_{1}\right)+\frac{\lambda_{S 2}}{2} S^{2}\left(H_{2}^{\dagger} H_{2}\right) .
$$

For suitable choices of parameters, these interactions allow for large enough DM annihilation cross section and as a result can accommodate the observed relic abundance.

After electroweak symmetry breaking three out of eight real degrees of freedom in $H_{1}$ and $H_{2}$ are absorbed as longitudinal components of $W^{ \pm}$and $Z$ bosons (for reviews see e.g. $[57,58])$. The remaining 5 degrees of freedom consist of two CP-even scalars $h$ and $H$,

$$
\left(\begin{array}{c}
H \\
h
\end{array}\right)=\left(\begin{array}{cc}
\cos \alpha & \sin \alpha \\
-\sin \alpha & \cos \alpha
\end{array}\right)\left(\begin{array}{l}
h_{1} \\
h_{2}
\end{array}\right),
$$

a CP-odd scalar $A \equiv-\chi_{1} \sin \beta+\chi_{2} \cos \beta$, and a pair of charged scalars $H^{ \pm} \equiv-H_{1}^{ \pm} \sin \beta+$ $H_{2}^{ \pm} \cos \beta$. Here $\tan \beta \equiv v_{2} / v_{1}$ is the ratio of $H_{2,1}$ condensates with $v_{E W} \equiv \sqrt{v_{1}^{2}+v_{2}^{2}}$. It is 
$h$ that we identify as the newly discovered particle with $125 \mathrm{GeV}$ mass. The interactions of the CP-even scalars, $h, H$, with the SM fermions and gauge bosons are given by

$$
\begin{aligned}
\mathcal{L} \supset & -\sum_{f=u, d, \ell}\left(\frac{r_{f} m_{f}}{v_{E W}} h+\frac{R_{f} m_{f}}{v_{E W}} H\right) \bar{f} f+g \sin (\beta-\alpha)\left(m_{W} W_{\mu}^{+} W^{\mu-}+\frac{m_{Z}}{2 c_{W}} Z_{\mu} Z^{\mu}\right) h \\
& +g \cos (\beta-\alpha)\left(m_{W} W_{\mu}^{+} W^{\mu-}+\frac{m_{Z}}{2 c_{W}} Z_{\mu} Z^{\mu}\right) H,
\end{aligned}
$$

with $r_{u}=\cos \alpha \csc \beta, r_{d}=r_{\ell}=-\sin \alpha \sec \beta, R_{u}=\sin \alpha \csc \beta, R_{d}=R_{\ell}=\cos \alpha \sec \beta$. After electroweak symmetry breaking there are also trilinear couplings of $h, H$ with the DM,

$$
\mathcal{L} \supset \frac{g_{S S h}}{2} v_{E W} h S^{2}+\frac{g_{S S H}}{2} v_{E W} H S^{2},
$$

where

$$
\begin{aligned}
g_{S S h} & =\lambda_{S 1} \sin \alpha \cos \beta-\lambda_{S 2} \cos \alpha \sin \beta, \\
g_{S S H} & =-\lambda_{S 1} \cos \alpha \cos \beta-\lambda_{S 2} \sin \alpha \sin \beta .
\end{aligned}
$$

DM annihilation into a pair of SM fermions, $S S \rightarrow \bar{f} f$, is mediated by both CP-even scalars, $h$ and $H$ and is proportional to $\sigma_{\text {ann }} \propto\left(g_{S S h} / m_{h}^{2}+g_{S S H} / m_{H}^{2}\right)^{2}$. For light DM the $g_{S S h}$ coupling also leads to $\mathcal{B}(h \rightarrow S S)$. As we show below the bounds on invisible decay width of the Higgs require $g_{S S h}<0.01$. Correct relic abundance then requires $g_{S S H} \sim \mathcal{O}(1)$, see figure 6.

Similarly, DM-nucleon scattering cross section also receives contributions from both $h$ and $H$ exchanges,

$$
\sigma_{p}^{S I}=\frac{m_{p}^{4}}{4 \pi\left(m_{\mathrm{DM}}+m_{p}\right)^{2} m_{H}^{4}}\left(\sum_{q} c_{q} f_{q}^{p}\right)^{2}
$$

where

$$
\begin{aligned}
c_{u, c, t} & =g_{S S h}\left(m_{H} / m_{h}\right)^{2} \cos \alpha \csc \beta+g_{S S H} \sin \alpha \csc \beta, \\
c_{d, s, b} & =-g_{S S h}\left(m_{H} / m_{h}\right)^{2} \sin \alpha \sec \beta+g_{S S H} \cos \alpha \sec \beta,
\end{aligned}
$$

while the relevant nuclear form factors $f_{q}^{p}$ are listed in eq. (A.7). The $h$ and $H$ contributions may interfere destructively. In fact, $\sigma_{p}^{S I}$ vanishes completely, if

$$
\frac{g_{S S h}}{g_{S S H}}=\frac{m_{h}^{2}}{m_{H}^{2}} \frac{\left(f_{u}^{p}+f_{c}^{p}+f_{t}^{p}\right) \sin \alpha \cos \beta+\left(f_{d}^{p}+f_{s}^{p}+f_{b}^{p}\right) \cos \alpha \sin \beta}{\left(-f_{u}^{p}-f_{c}^{p}-f_{t}^{p}\right) \cos \alpha \cos \beta+\left(f_{d}^{p}+f_{s}^{p}+f_{b}^{p}\right) \sin \alpha \sin \beta} .
$$

Note that it is possible to fulfill this requirement even if $g_{S S h}=0$. Then $\mathcal{B}(h \rightarrow S S)=0$, while eq. (4.17) gives

$$
\frac{\tan \alpha}{\tan \beta}=-\frac{f_{d}^{p}+f_{s}^{p}+f_{b}^{p}}{f_{u}^{p}+f_{c}^{p}+f_{t}^{p}}
$$

As we will show below the pseudo-decoupling limit, $\beta-\alpha=\pi / 2$, where the couplings of the Higgs to $W$ and $Z$ are the SM ones, cf. eq. (4.12), is preferred by recent Higgs data. 


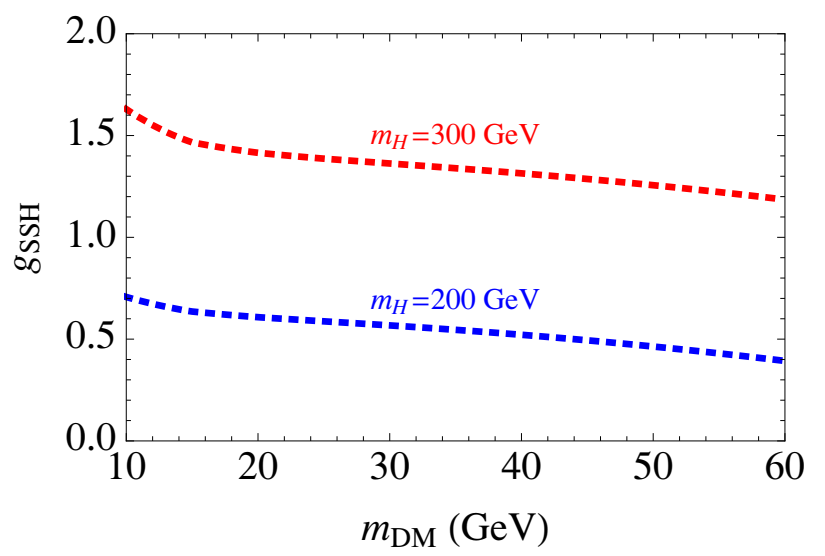

Figure 6. The value of $g_{S S H}$ that gives the observed DM relic density in 2HDM-II models with extra singlet, as function of DM mass, $m_{\mathrm{DM}}$, for the case where the invisible decay width of the Higgs and the DM-proton scattering cross section both vanish. Two choices of the heavy CP-even Higgs mass, $m_{H}=200,300 \mathrm{GeV}$ are shown.

In this limit eq. (4.18) then completely fixes the value of $\tan \beta$; i.e., using the values of nuclear form factors in eq. (A.7) one obtains $\tan \beta \simeq 0.61$.

In the limit where $\mathcal{B}(h \rightarrow S S)$ vanishes, the relic abundance is set by DM annihilation with the heavy CP-even Higgs boson $H$ in the $s$-channel. In figure 6 , we plot the coupling $g_{S S H}$ giving the correct relic abundance as a function of DM mass, $m_{\mathrm{DM}}$, for two sample values of heavy CP-even Higgs boson masses, $m_{H}=200,300 \mathrm{GeV}$. We also set $\tan \beta=0.61$ such that $\sigma_{p}^{S I}$ vanishes. For heavier $H$ a larger value of $g_{S S H}$ coupling is needed. Perturbativity therefore bounds $m_{H}$ from above, with $m_{H} \lesssim 850 \mathrm{GeV}$ for $g_{S H H} \lesssim 4 \pi$ (and $m_{H} \lesssim 450 \mathrm{GeV}$ for $g_{S H H} \lesssim 4$ ). Note that in this case $H$ decays invisibly practically $100 \%$ of the time. In principle $H$ can be directly searched in the process of associated production with a $Z$ boson (see, e.g., a recent ATLAS analysis of $p p \rightarrow Z h \rightarrow l^{+} l^{-}$invisible [59]). The challenge is that in the limit $\beta-\alpha=\pi / 2$, the couplings of $H$ to gauge bosons vanish. As a result, the heavy Higgs boson in this scenario can easily escape such collider searches. On the other hand, $H$ also couples to SM fermions with roughly SM strengths, thus making $g g \rightarrow H(t \bar{t})$ the dominant production mechanisms at the LHC. Especially in the second case, the dominant decay mode $H \rightarrow \mathrm{DM}+\mathrm{DM}$ then leads to the interesting $t \bar{t}+E_{T}^{\text {miss }}$ signature. For $m_{H}=200,300 \mathrm{GeV}$, we find using [60] the cross-section estimates of $\sigma_{t \bar{t} E_{T}^{\text {miss }}}=29 \mathrm{fb}, 7.7 \mathrm{fb}$ at $8 \mathrm{TeV}$ and $\sigma_{t \bar{t} E_{T}^{\text {miss }}}=150 \mathrm{fb}, 51 \mathrm{fb}$ at $14 \mathrm{TeV}$ LHC, respectively. Given these small cross-sections, also compared to irreducible SM $(t \bar{t}+Z)$ backgrounds [61-63], the search remains challenging for the foreseeable future. On the other hand, interesting mono-jet plus missing transverse energy signature would come from $g g \rightarrow H+$ jet. Using this particular signature, a dedicated analysis for the SM Higgs boson invisible decay was performed in [64]. The upper limit on $\mu_{H j} \equiv \sigma_{g g \rightarrow H j} \times \mathcal{B}(H \rightarrow$ inv $) / \sigma_{g g \rightarrow H j}^{\mathrm{SM}}$ at $95 \%$ C.L. was found to be $\mu_{H j}<25(50)$ for $200 \mathrm{GeV}(300 \mathrm{GeV})$ Higgs boson using just $1 \mathrm{fb}^{-1}$ of data at $7 \mathrm{TeV}$. It might be possible for $14 \mathrm{TeV}$ LHC to probe the prediction of this model, $\mu_{H j}=R_{u}^{2}=2.7$. 


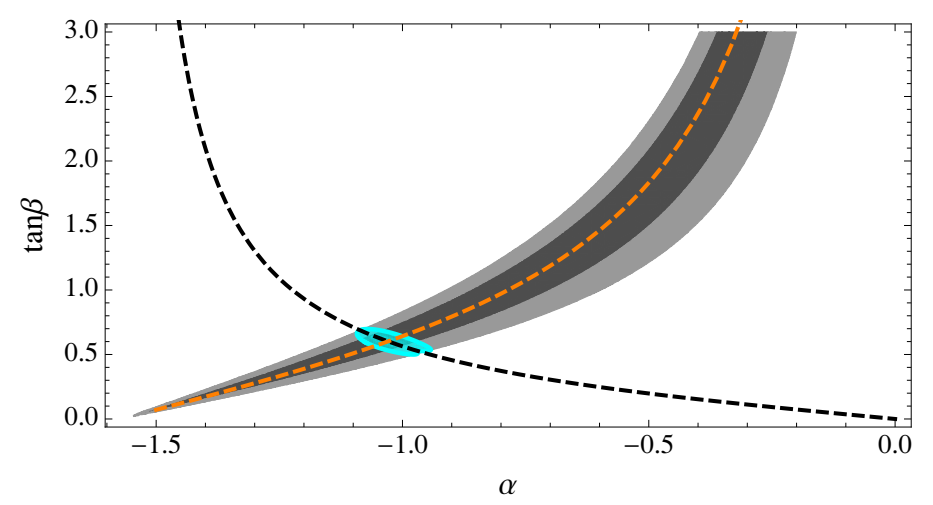

Figure 7. The $68.3 \%$ and $95.5 \%$ C.L. parameter regions in 2 HDM-II with an extra singlet that are allowed by the Higgs signal strength data are shown in dark grey and light grey, respectively. Orange-dashed curve correspond to $\beta-\alpha=\pi / 2$. Black-dashed curve correspond to eq. (4.18). The $95.5 \%$ C.L. region allowed by the Higgs data together with direct DM detection bound from XENON100 is shown in cyan. For definiteness we assume $m_{H}=200 \mathrm{GeV}, m_{S}=40 \mathrm{GeV}$ and $g_{S S H}$ such that the proper DM thermal relic is obtained.

Finally, we assess the quantitative impact of existing Higgs measurements on the model's parameter space by performing a fit to the latest LHC Higgs data assuming that $h$ is the newly discovered Higgs resonance (for details see appendix B). The partial decay widths normalized to the SM ones are given by

$$
\begin{array}{rlrl}
\frac{\Gamma_{h \rightarrow W W, Z Z}}{\Gamma_{h \rightarrow W W, Z Z}^{\mathrm{SM}}} & =\sin ^{2}(\beta-\alpha) \equiv r_{V}^{2}, & & \frac{\Gamma_{h \rightarrow b b}}{\Gamma_{h \rightarrow b b}^{\mathrm{SM}}}=r_{d}^{2}, \\
\frac{\Gamma_{h \rightarrow \tau \tau}}{\Gamma_{h \rightarrow \tau \tau}^{\mathrm{SM}}}=r_{l}^{2}, & & \frac{\Gamma_{h \rightarrow \gamma \gamma}}{\Gamma_{h \rightarrow \gamma \gamma}^{\mathrm{SM}}}=\left|-1.28 r_{V}+0.283 r_{u}\right|^{2},
\end{array}
$$

while the normalized production rates are

$$
\frac{\sigma_{g g F}}{\sigma_{g g F}^{\mathrm{SM}}}=\left|1.06 r_{u}+(-0.06+\imath 0.09) r_{d}\right|^{2}, \quad \frac{\sigma_{V B F+V H}}{\sigma_{V B F+V H}^{\mathrm{SM}}}=r_{V}^{2} .
$$

In the Higgs signal strengths, $\mu_{i}$, one measures the product of cross section and Higgs branching ratios. Therefore in all the signal strengths the total Higgs decay width enters. This can be modified by the invisible decay width of the Higgs, and as a result one is quite sensitive to it. Normalized to the SM the total width is given by

$$
\hat{\Gamma} \equiv \frac{\Gamma_{\text {total }}}{\Gamma_{\text {total }}^{\mathrm{SM}}}=\frac{0.569 r_{d}^{2}+0.252 r_{V}^{2}+0.063 r_{l}^{2}+0.085 \frac{\sigma_{g g F}}{\sigma_{g g F}^{\mathrm{SM}}}+0.026 r_{u}^{2}}{1-\mathcal{B}(h \rightarrow S S)} .
$$

Numerical values for loop functions in $h \rightarrow \gamma \gamma$ and $h \rightarrow g g$ are taken from [65], while $\mathrm{SM}$ branching ratios for $m_{h}=125 \mathrm{GeV}$ Higgs boson are taken from [66]. In our model all the Higgs signal strengths $\mu_{i}$ depend on three parameters, $\alpha, \beta$ and $\mathcal{B}(h \rightarrow S S)$. Figure 7 shows the $68.3 \%$ and $95.5 \%$ C.L. allowed region in the parameter space $(\alpha, \tan \beta)$ obtained from a global fit after marginalizing over $\mathcal{B}(h \rightarrow S S)$. The allowed parameter 
space is constrained to a very narrow region around $\beta-\alpha=\pi / 2$. We also derive the bound on invisible branching ratio of the Higgs by marginalizing over $\alpha$ and $\tan \beta$. We get $\mathcal{B}(h \rightarrow S S)<0.3$ at $95.5 \%$ C.L., which implies that $g_{S S h}<0.01$ for DM mass up to $m_{h} / 2$. We emphasize that $\mathcal{B}(h \rightarrow$ invisible $)$ is a free parameter in this model, and can be both close to present experimental bound or much smaller, depending on the derived dimensionless parameter $g_{S S h}$.

Finally, we combine the Higgs data and 90\% C.L. upper bound on spin-independent DM-nucleon cross section from XENON100 [31] into a single $\chi^{2}$. For illustration we fix $m_{H}=200 \mathrm{GeV}, m_{S}=40 \mathrm{GeV}$ and $g_{S S H}$ to value determined by relic density. The DM scattering cross section $\sigma_{p}^{S I}$ and the signal strength rates $\mu_{i}$ are expressed in terms of three fitting parameters $\alpha, \beta$ and $g_{S S h}$. After marginalizing over $g_{S S h}$, we obtain the $95.5 \%$ C.L. allowed region in $(\alpha, \tan \beta)$ plane, shown as cyan region in figure 7. Marginalizing analogously over $\alpha$ and $g_{S S h}$, we find $\tan \beta=(0.61 \pm 0.03)$.

\section{3 $\mathrm{SM}+\mathrm{DM}$ with extra scalar singlet}

In our final example of a viable Higgs portal model of DM we add to the SM two real scalars, $\phi$ and $S$ (for existing studies of similar models see $[67,68]$ ). Under the SM gauge group both scalars therefore transform as

$$
\phi \sim(1,1,0), \quad S \sim(1,1,0)
$$

The singlet $S$ is the DM candidate, odd under $Z_{2}$, while $\phi$ is even. The resulting scalar potential is

$$
\begin{aligned}
V= & m_{H}^{2} H^{\dagger} H+\frac{m_{2}^{2}}{2} \phi^{2}+\frac{m_{3}^{2}}{2} S^{2}+\kappa m_{2}^{3} \phi+\frac{\lambda_{1}}{2}\left(H^{\dagger} H\right)^{2}+\frac{\lambda_{2}}{8} \phi^{4}+\frac{\lambda_{3}}{8} S^{2} \\
& +\frac{\lambda_{4}}{2} H^{\dagger} H \phi^{2}+\frac{\lambda_{5}}{2} H^{\dagger} H S^{2}+\frac{\lambda_{6}}{4} \phi^{2} S^{2}+\frac{\mu_{1}}{2} \phi^{3}+\mu_{2} H^{\dagger} H \phi+\frac{\mu_{3}}{2} S^{2} \phi,
\end{aligned}
$$

while the Yukawa interactions take the usual form

$$
-\mathcal{L}_{Y}=Y_{u} \bar{Q} \tilde{H} u_{R}+Y_{d} \bar{Q} H d_{R}+Y_{\ell} \bar{L} H e_{R}+\text { h.c. } .
$$

For simplicity, we assume that $\phi$ does not acquire a vacuum expectation value by appropriately adjusting the parameter $\kappa$ (this has no relevant phenomenological consequences apart from simplifying our discussion). The scalar mass matrix is given by

$$
M_{\mathrm{sc}}^{2}=\left(\begin{array}{cc}
m_{h}^{2} & \mu_{2} v_{E W} \\
\mu_{2} v_{E W} & m_{\phi}^{2}
\end{array}\right),
$$

where $m_{h}^{2}=\lambda_{1} v_{E W}^{2}$ and $m_{\phi}^{2}=m_{2}^{2}+\lambda_{4} v_{E W}^{2} / 2$. Parameter $\mu_{2}$ induces mixing between $h$ and $\phi$, so that the physical neutral scalars $h_{1}, h_{2}$ are given by

$$
\begin{aligned}
& h_{1}=h \cos \alpha+\phi \sin \alpha, \\
& h_{2}=-h \sin \alpha+\phi \cos \alpha,
\end{aligned}
$$




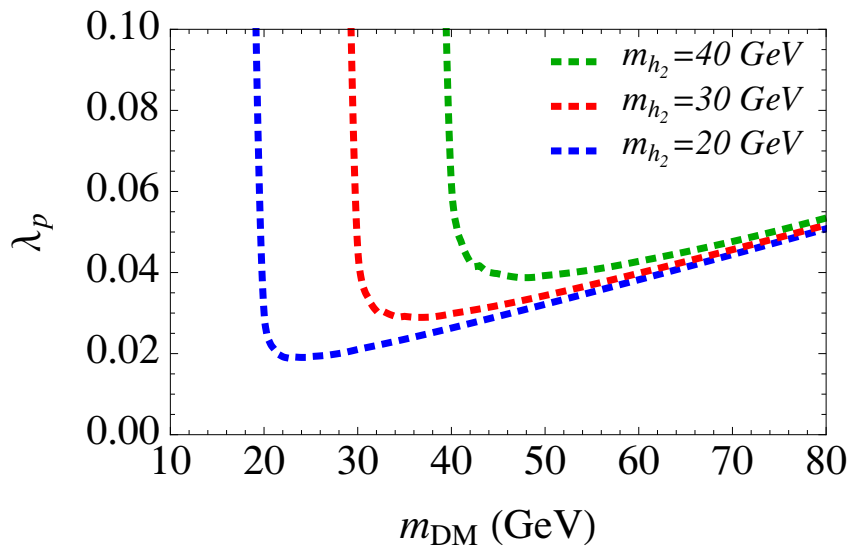

Figure 8. Coupling $\lambda_{p}$ for which the proper relic abundance is obtained in the model with an extra scalar singlet (4.23).

with the mixing angle given by

$$
\tan 2 \alpha=\frac{2 \mu_{2} v_{E W}}{m_{h}^{2}-m_{\phi}^{2}} .
$$

We will assume that $m_{h_{1}} / 2>m_{S}>m_{h_{2}}$ with $m_{h_{1}}=125 \mathrm{GeV}$.

The couplings of $h_{1}\left(h_{2}\right)$ to the SM fields are the same as for the SM Higgs boson except that they are rescaled by $\cos \alpha(\sin \alpha)$. The mixing angle $\alpha$ has been constrained by LEP [69], so that at $95 \%$ C.L. $|\sin \alpha|<0.13$ for $m_{h_{2}}=20 \mathrm{GeV}$ and $|\sin \alpha|<0.2$ for $m_{h_{2}}=50 \mathrm{GeV}$. On the other hand, $\sin \alpha$ also has to be greater than $10^{-8}$, otherwise $h_{2}$ is sufficiently long lived that it escapes the detector. For $\sin \alpha \sim 10^{-4}$ the $h_{2}$ particle travels less than a few $\mu \mathrm{m}$ before decaying and can be searched for using displaced vertices. Note that the branching ratios of $h_{2}$ are not affected by $\sin \alpha$ and are the same as they would be for the SM Higgs with $m_{h_{2}}$ mass. For instance, for $m_{h_{2}}=20 \mathrm{GeV}$ the dominant branching ratio is $\mathcal{B}\left(h_{2} \rightarrow b \bar{b}\right) \sim 85 \%$.

The relic abundance is set by the dominant DM annihilation process $S S \rightarrow h_{2} h_{2}$, with the annihilation cross section given by

$$
\sigma_{S S \rightarrow h_{2} h_{2}}=\frac{\lambda_{p}^{2}}{32 \pi s} \frac{\sqrt{1-4 m_{h_{2}}^{2} / s}}{\sqrt{1-4 m_{S}^{2} / s}},
$$

where $\lambda_{p}=\lambda_{6} \cos ^{2} \alpha+\lambda_{5} \sin ^{2} \alpha$. The values of $\lambda_{p}$ for which the correct relic abundance is obtained are shown in figure 8 as a function of DM mass, $m_{\mathrm{DM}}$, for three choices of light scalar mass $m_{h_{2}}$. Note that $\lambda_{p}$ that governs the relic abundance is different from $\lambda_{h}=\lambda_{5} \cos \alpha-\lambda_{6} \sin \alpha$ that governs the invisible Higgs branching ratio, $\mathcal{B}(h \rightarrow$ invisible). The relic abundance and invisible decay width of the Higgs are thus decoupled in this Higgs portal model.

Next, we perform fit to the latest available LHC Higgs data. Unlike the 2HDM-II case, section 4.2 , here all the Higgs $\left(h_{1}\right)$ signal strengths are rescaled by common factor 


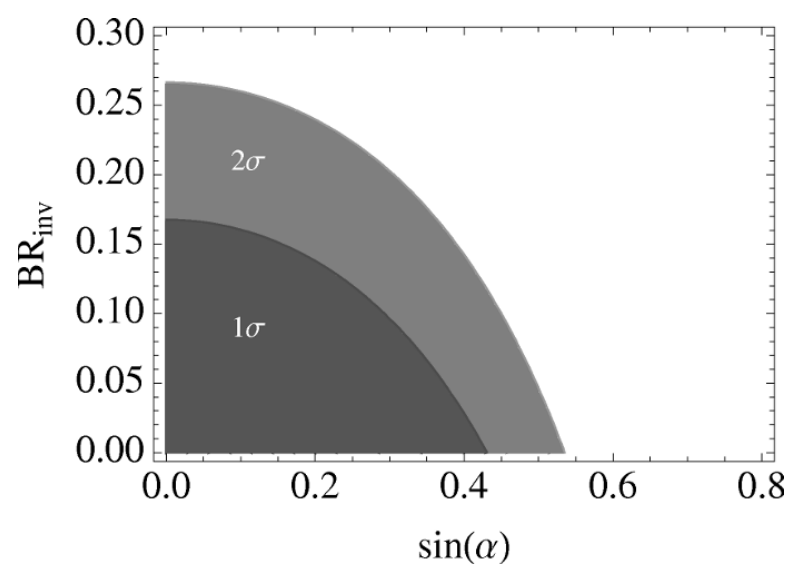

Figure 9. Constraints from Higgs signal strengths of the Higgs portal model of light DM with an extra singlet. $1 \sigma$ and $2 \sigma$ constraints on $\sin \alpha$ and $\mathcal{B}(h \rightarrow$ invisible $)$ are show as dark and lighter grey regions, respectively.

$\cos ^{2} \alpha$. Also, there are additional contributions to the total Higgs decay width coming from $h_{1} \rightarrow h_{2} h_{2}$ and $h_{1} \rightarrow S S$. The Higgs signal strengths, therefore, are given by

$$
\mu^{h \rightarrow S M}=\cos ^{2} \alpha(1-\Delta \hat{\mathcal{B}}),
$$

with $\Delta \hat{\mathcal{B}} \equiv \mathcal{B}\left(h_{1} \rightarrow S S\right)+\mathcal{B}\left(h_{1} \rightarrow h_{2} h_{2}\right)$. A direct bound on invisible Higgs decay width from ATLAS analysis of $p p \rightarrow Z h \rightarrow l^{+} l^{-}$invisible [59], is added to $\chi^{2}$ as

$$
\chi_{i n v}^{2}=\left(\frac{\cos ^{2} \alpha \mathcal{B}\left(h_{1} \rightarrow S S\right)+0.19}{0.43}\right)^{2},
$$

assuming that $\sin \alpha \gtrsim 10^{-4}$ so that $h_{2}$ decays instantaneously. We then take $\sin \alpha, \mathcal{B}\left(h_{1} \rightarrow\right.$ $S S)$ and $\mathcal{B}\left(h_{1} \rightarrow h_{2} h_{2}\right)$ as fitting parameters. We obtain $95.5 \%$ C.L. bounds on each parameter to be $|\sin \alpha|<0.5, \mathcal{B}\left(h_{1} \rightarrow h_{2} h_{2}\right)<0.24$ and $\mathcal{B}\left(h_{1} \rightarrow S S\right)<0.22$. Note that the bound on $\sin \alpha$ obtained from this fit is less stringent than the LEP limit. In figure 9 , we show $68.3 \%$ and $95.5 \%$ C.L. allowed region in the parameter space of $\sin \alpha$ and $\mathcal{B}\left(h_{1} \rightarrow S S\right)$, after marginalizing over $\mathcal{B}\left(h_{1} \rightarrow h_{2} h_{2}\right)$. If $\sin \alpha$ is very small, so that $h_{2}$ escapes the detector, then we obtain $\mathcal{B}\left(h_{1} \rightarrow\right.$ invisible $)<0.22$.

Since there is an extra light scalar state, there are interesting collider signatures beside the invisible decay width of the Higgs. The Higgs can also decay to two light scalars, $h_{1} \rightarrow h_{2} h_{2}$, where $h_{2}$ decays to $b \bar{b}$ pairs. These decay chains can then be searched for using associated $h Z$ or $h W$ production with four b-tagged jets in the final state (possibly originating from two displaced secondary vertices, see also [70]) combining to the Higgs mass. As discussed above, the $h_{1} \rightarrow h_{2} h_{2}$ branching ratio can be sizeable, of $\mathcal{O}(20 \%)$.

\section{Conclusions}

In this paper we have extended the analysis of Higgs portal models of DM by including higher dimensional operators. We focused on the case where DM is light, so that $h \rightarrow$ 
DM+DM decays are kinematically allowed. The main difference between the minimal Higgs portals and the case where higher dimensional operators dominate, is that there is now a new scale $\Lambda$ in the problem. In fact, already for minimal Higgs portal with fermionic DM one is forced to introduce a dimensionful scale $\Lambda$ since the Higgs couplings then require at least dimension 5 operators. We arrive at the following general conclusions

- First assume that an EFT description of SM+DM as the only weak scale dynamical degrees of freedom is valid and all dimensionless coefficients are $\mathcal{O}(1)$. If $h \rightarrow$ $\mathrm{DM}+\mathrm{DM}$ is discovered close to its present experimental limit, at the order of $\mathcal{O}$ (few $10 \%$ ), then DM cannot be a thermal relic, or its relic density must be controlled by interactions not involving the Higgs field.

- Higgs portal to DM is still possible if either EFT is not valid or if $\mathcal{B}(h \rightarrow$ invisible) is suppressed below naive counting estimate (or both). In both cases there need to be other light particles, with masses below $\mathcal{O}($ few $100 \mathrm{GeV})$.

We demonstrate this with three examples of viable Higgs portal models of light DM, (i) the SM extended by DM scalar along with electroweak triplet and singlet (subsection 4.1), (ii) a Two Higgs Doublet Model of type II with an addition of scalar DM field (subsection 4.2), (iii) SM with DM and an extra scalar singlet that is lighter than DM (subsection 4.3). All the examples share the feature that the invisible Higgs branching ratio and the relic abundance are decoupled and are governed by different parameters. Furthermore, only in example (ii) the dominant DM annihilation channel is to $b \bar{b}$ pairs as in the simplest Higgs portal models. As a result this model also requires tuned cancellation to avoid direct DM detection constraints.

Since the Higgs portals of DM require additional light particles, there may be interesting phenomenological consequences. Indeed, non-trivial dynamics taking place below the $\mathrm{TeV}$ could leave significant footprints in low energy precision observables, or could be directly detected at high-energy collider experiments. For instance, the charged scalars in example (i) can lead to lepton flavor violating decays, in example (ii) the heavy Higgs decay is dominated by the invisible channels, while in (iii) the Higgs decays to four $b$ jets at the level of a few tens of percent are possible.

\section{Acknowledgments}

A.G., J.J. and J.F.K were supported in part by the Slovenian Research Agency. J.Z. was supported in part by the U.S. National Science Foundation under CAREER Grant PHY-1151392.

\section{A Relic density and direct detection}

The DM relic abundance is found by solving the following Boltzmann equation,

$$
\frac{d Y}{d x}=\frac{1}{3 H} \frac{d s}{d x}\langle\sigma v\rangle\left(Y^{2}-Y_{\mathrm{eq}}^{2}\right)
$$


where $H$ is the Hubble constant, $x \equiv m_{\mathrm{DM}} / T$ with $m_{\mathrm{DM}}$ the DM mass, and $Y \equiv n / s$ with $n$ and $s$ the number density and entropy density respectively. The thermal average of the annihilation cross section is given by [71]

$$
\langle\sigma v\rangle=\int_{\epsilon_{t h}}^{\infty} d \epsilon \frac{2 x}{K_{2}(x)^{2}} \sqrt{\epsilon}(1+2 \epsilon) K_{1}(2 x \sqrt{1+\epsilon}) \sigma v
$$

Here $K_{i}(x)$ is the $i$-th order modified Bessel function of the second kind. The parameter $\epsilon$ is the kinetic energy per unit mass defined as $\epsilon \equiv\left(s-4 m_{\mathrm{DM}}^{2}\right) /\left(4 m_{\mathrm{DM}}^{2}\right)$, while $\epsilon_{t h}$ is the threshold kinetic energy per unit mass. It is $\epsilon_{t h}=0$ if $2 m_{\mathrm{DM}} \geq m_{3}+m_{4}$, and $\epsilon_{t h}=\left(m_{3}+m_{4}\right)^{2} /\left(4 m_{\mathrm{DM}}^{2}\right)-1$ if $2 m_{\mathrm{DM}}<m_{3}+m_{4}$, with $m_{3}$ and $m_{4}$ the masses of the final state particles.

In the early universe, DM is assumed to be in equilibrium. Once the temperature drops below the DM mass, $Y_{\text {eq }}$ is exponentially suppressed. When the freeze-out temperature is reached, the equilibrium is no longer maintained. As the result, one can integrate the Boltzmann equation to determine relic abundance [71, 72]

$$
\Omega h^{2}=\frac{1.07 \times 10^{9} \mathrm{GeV}^{-1}}{\sqrt{g_{*}} M_{\mathrm{Pl}}}\left(\int_{x_{f}}^{\infty} d x \frac{\langle\sigma v\rangle}{x^{2}}\right)^{-1},
$$

where $M_{\mathrm{Pl}}=1.22 \times 10^{19} \mathrm{GeV}$ is the Planck mass, and $g_{*}$ is the number of effective relativistic degrees of freedom at freeze-out. The freeze-out temperature $T_{f}$ is determined through $\left(x_{f} \equiv m_{\mathrm{DM}} / T_{f}\right)$

$$
x_{f}=\ln \frac{0.038 g M_{\mathrm{Pl}} m_{\mathrm{DM}}\langle\sigma v\rangle}{\sqrt{g_{*} x_{f}}},
$$

with $g$ the number of DM degrees of freedom.

We review next the calculation of direct DM detection bounds. The operators given in eqs. (3.2) and (3.4) lead to the DM-quark interactions which then induce the scattering of DM on nuclei. For operators in eq. (3.2), the DM-nucleon cross sections are found to be $(N=p, n)$

$$
\begin{aligned}
\sigma_{p, n}^{\phi S I} & =\frac{8 G_{F}^{2}}{\pi} c_{\phi}^{2}\left(\frac{v_{E W}}{\Lambda}\right)^{4} \mu_{\phi N}^{2}\left(2 Y_{u, d}+Y_{d, u}\right)^{2} \\
\sigma_{p, n}^{\psi S I} & =\frac{G_{F}^{2}}{2 \pi}\left(c_{\psi}^{L}+c_{\psi}^{R}\right)^{2}\left(\frac{v_{E W}}{\Lambda}\right)^{4} \mu_{\psi N}^{2}\left(2 Y_{u, d}+Y_{d, u}\right)^{2} \\
\sigma_{p, n}^{\psi S D} & =\frac{3 G_{F}^{2}}{8 \pi}\left(c_{\psi}^{L}-c_{\psi}^{R}\right)^{2}\left(\frac{v_{E W}}{\Lambda}\right)^{4} \mu_{\psi N}^{2}\left(-\Delta_{u}^{p, n}+\Delta_{d}^{p, n}+\Delta_{s}^{p, n}\right)^{2}, \\
\sigma_{p, n}^{V S I} & =\frac{32 G_{F}^{2}}{\pi} c_{V}^{2}\left(\frac{v_{E W}}{\Lambda}\right)^{4} \mu_{\psi N}^{2}\left(2 Y_{u, d}+Y_{d, u}\right)^{2} .
\end{aligned}
$$


Similarly, for operators in eq. (3.4) we have

$$
\begin{aligned}
\sigma_{N}^{\phi S I} & =\frac{1}{8 \pi} \frac{\mu_{\phi N}^{2} m_{N}^{2} v_{E W}^{2}}{\Lambda^{4}} \frac{1}{m_{\phi}^{2}}\left(\sum_{q} f_{\phi} \frac{f_{q}^{N}}{m_{q}}\right)^{2}, \\
\sigma_{N}^{\psi S I} & =\frac{1}{2 \pi} \frac{\mu_{\psi N}^{2} m_{N}^{2} v_{E W}^{2}}{\Lambda^{6}}\left[\left(\sum_{q} f_{\psi}^{S} \frac{f_{q}^{N}}{m_{q}}\right)^{2}+\frac{1}{2} \frac{|\boldsymbol{p}|^{2}}{m_{\psi}^{2}}\left(\sum_{q} f_{\psi}^{P} \frac{f_{q}^{N}}{m_{q}}\right)^{2}\right], \\
\sigma_{N}^{\psi S D} & =\frac{6}{\pi} \frac{\mu_{\psi N}^{2} v_{E W}^{2}}{\Lambda^{6}}\left(\sum_{q} f_{\psi}^{T} \delta_{q}^{N}\right)^{2}, \\
\sigma_{N}^{V S I} & =\frac{1}{2 \pi} \frac{\mu_{V N}^{2} m_{N}^{2} v_{E W}^{2}}{\Lambda^{4}} \frac{1}{M_{V}^{2}}\left(f_{V}\right)^{2}\left(\sum_{q} f_{V} \frac{f_{q}^{N}}{m_{q}}\right)^{2} .
\end{aligned}
$$

In above equations, $|\boldsymbol{p}| \sim 1 \mathrm{MeV}$ is the DM momentum in the center of mass frame, $\mu_{\chi N}$ is the DM-nucleon reduced masses (with $\chi=\phi, \psi, V$ ), and the relevant quark- $Z$ couplings are $Y_{u}=\frac{1}{2}-\frac{4}{3} s_{W}^{2}$, and $Y_{d}=-\frac{1}{2}+\frac{2}{3} s_{W}^{2}$. The parameters $f_{q}^{N} \equiv m_{N}^{-1}\left\langle N\left|m_{q} \bar{\psi}_{q} \psi_{q}\right| N\right\rangle, \Delta_{q}^{N}$, and $\delta_{q}^{N}$ indicate the nucleon form factors for scalar, axial-vector, and tensor interactions, respectively. Their values are given by [42]

$$
\begin{aligned}
& f_{u}^{p}=0.023, \\
& f_{d}^{p}=0.033, \quad f_{s}^{p}=0.26, \\
& f_{u}^{n}=0.018, \\
& f_{d}^{n}=0.042, \quad f_{s}^{n}=0.26, \\
& f_{c, b, t}^{p, n}=\frac{2}{27}\left(1-\sum_{q=u, d, s} f_{q}^{p, n}\right), \\
& \Delta_{u}^{p, n}=0.842, \\
& \Delta_{d}^{p, n}=-0.427, \\
& \Delta_{s}^{p, n}=-0.085, \\
& \delta_{u}^{p, n}=0.84, \\
& \delta_{d}^{p, n}=-0.23, \\
& \delta_{s}^{p, n}=-0.05 .
\end{aligned}
$$

We use XENON100 bounds from ref. [31] for spin-independent (SI) case and ref. [40] for spin-dependent (SD) case to constrain the parameter space given by the relic density. We always use the more constraining choice.

\section{B Analysis of Higgs data}

In our fitting procedures we follow the method adopted in references [73-76]. The latest available LHC Higgs data are presented in table 2. Measurements are reported in terms of signal strengths normalized to the SM predictions

$$
\mu_{(k)}^{i}=\frac{\sigma_{(k)}}{\sigma_{(k)}^{\mathrm{SM}}} \frac{\mathcal{B}_{i}}{\mathcal{B}_{i}^{\mathrm{SM}}}
$$

where index $i$ represents the decay mode, while $k$ denotes different production channels. ATLAS and CMS also combine different production sub-channels for a given decay mode to provide separation into production mechanisms. Results are presented in $2 \mathrm{D}$ plots in which gluon-gluon fusion ( $\mathrm{ggF}$ ) and associated production with a top pair (ttH) are combined as one signal $\left(\mu_{(g g F+t t H)}\right)$, while vector boson fusion (VBF) and associated production 


\begin{tabular}{|c|c|c|c|}
\hline Decay channel & Production mode & Signal strength & Correlation \& Reference \\
\hline \multicolumn{4}{|c|}{ ATLAS } \\
\hline$h \rightarrow b \bar{b}$ & $\mathrm{VH}$ & $-0.4 \pm 1.0$ & {$[8]$} \\
\hline \multirow{2}{*}{$h \rightarrow Z Z^{*}$} & $\operatorname{ggF}+\mathrm{ttH}$ & $1.51 \pm 0.52$ & \multirow{2}{*}{$\rho=-0.5,[3,8]$} \\
\hline & $\mathrm{VBF}+\mathrm{VH}$ & $2.0 \pm 2.1$ & \\
\hline \multirow{2}{*}{$h \rightarrow W W^{*}$} & $\operatorname{ggF}+\mathrm{ttH}$ & $0.79 \pm 0.35$ & \multirow{2}{*}{$\rho=-0.2,[8,77]$} \\
\hline & $\mathrm{VBF}+\mathrm{VH}$ & $1.72 \pm 0.77$ & \\
\hline \multirow{2}{*}{$h \rightarrow \gamma \gamma$} & $\operatorname{ggF}+\mathrm{ttH}$ & $1.61 \pm 0.41$ & \multirow{2}{*}{$\rho=-0.25,[8,78]$} \\
\hline & $\mathrm{VBF}+\mathrm{VH}$ & $1.95 \pm 0.82$ & \\
\hline \multirow{2}{*}{$h \rightarrow \tau \tau$} & $\operatorname{ggF}+\mathrm{ttH}$ & $2.3 \pm 1.6$ & \multirow{2}{*}{$\rho=-0.5,[8]$} \\
\hline & $\mathrm{VBF}+\mathrm{VH}$ & $-0.2 \pm 1.1$ & \\
\hline \multicolumn{2}{|c|}{$p p \rightarrow Z h \rightarrow l^{+} l^{-} \mathrm{inv}$} & $\mathrm{BR}_{\text {inv }}=-0.19 \pm 0.43$ & {$[59,79]$} \\
\hline \multicolumn{4}{|c|}{ CMS } \\
\hline \multirow{3}{*}{$h \rightarrow b \bar{b}$} & $\mathrm{VH}$ & $1.0 \pm 0.5$ & {$[80]$} \\
\hline & VBF & $0.7 \pm 1.4$ & {$[81]$} \\
\hline & $\mathrm{ttH}$ & $0.6 \pm 2.6$ & {$[82]$} \\
\hline \multirow{2}{*}{$h \rightarrow W W^{*}$} & $\operatorname{ggF}+\mathrm{ttH}$ & $0.76 \pm 0.23$ & \multirow{2}{*}{$\rho=-0.2,[9]$} \\
\hline & $\mathrm{VBF}+\mathrm{VH}$ & $0.35 \pm 0.69$ & \\
\hline \multirow{2}{*}{$h \rightarrow Z Z^{*}$} & $\operatorname{ggF}+\mathrm{ttH}$ & $0.90 \pm 0.45$ & \multirow{2}{*}{$\rho=-0.7,[9,83]$} \\
\hline & $\mathrm{VBF}+\mathrm{VH}$ & $1.0 \pm 2.3$ & \\
\hline \multirow{2}{*}{$h \rightarrow \gamma \gamma$} & $\operatorname{ggF}+\mathrm{ttH}$ & $0.48 \pm 0.39$ & \multirow{2}{*}{$\rho=-0.48,[9,84]$} \\
\hline & $\mathrm{VBF}+\mathrm{VH}$ & $1.70 \pm 0.88$ & \\
\hline \multirow{2}{*}{$h \rightarrow \tau \tau$} & $\operatorname{ggF}+\mathrm{ttH}$ & $0.68 \pm 0.80$ & \multirow{2}{*}{$\rho=-0.46,[9]$} \\
\hline & $\mathrm{VBF}+\mathrm{VH}$ & $1.61 \pm 0.83$ & \\
\hline
\end{tabular}

Table 2. The LHC Higgs data used in the analysis, with the Higgs decay channel, production mode, the signal strength normalized to the SM and the correlation coefficient (for details see text).

with a gauge boson $(\mathrm{VH})$ as another, $\left(\mu_{(V B F+V H)}\right)$. In this case, we parametrize the likelihood with

$$
\chi_{1}^{2}=\sum_{i}\left(\begin{array}{c}
\mu_{(g g F+t t H)}^{i}-\hat{\mu}_{(g g F+t t H)}^{i} \\
\mu_{(V B F+V H)}^{i}-\hat{\mu}_{(V B F+V H)}^{i}
\end{array}\right)^{T} V_{i}^{-1}\left(\begin{array}{c}
\mu_{(g g F+t t H)}^{i}-\hat{\mu}_{(g g F+t t H)}^{i} \\
\mu_{(V B F+V H)}^{i}-\hat{\mu}_{(V B F+V H)}^{i}
\end{array}\right),
$$

where the correlation matrices are given by

$$
V_{i}=\left(\begin{array}{cc}
\left(\hat{\sigma}_{(g g F+t t H)}^{i}\right)^{2} & \rho^{i} \hat{\sigma}_{(g g F+t t H)}^{i} \hat{\sigma}_{(V B F+V H)}^{i} \\
\rho^{i} \hat{\sigma}_{(g g F+t t H)}^{i} \hat{\sigma}_{(V B F+V H)}^{i} & \left(\hat{\sigma}_{(V B F+V H)}^{i}\right)^{2}
\end{array}\right) .
$$

Best-fit values $(\hat{\mu})$, variances $(\hat{\sigma})$ and correlations $(\rho)$ are obtained from the plots provided by the experiments and listed in table 2 . 
Other data are given in terms of signal strengths with specified production mechanism. In this case, we parametrize the likelihood with

$$
\chi_{2}^{2}=\sum_{i}\left(\frac{\mu_{i}-\hat{\mu}_{i}}{\hat{\sigma}_{i}}\right)^{2} .
$$

The total $\chi^{2}$ function is given by the sum of all the contributions. In order to confront the DM model to the data, we express all signal strengths $(\mu)$ in terms of model parameters and minimize $\chi^{2}$ to find the best fit point. The best fit regions are defined by appropriate cumulative distribution functions.

Open Access. This article is distributed under the terms of the Creative Commons Attribution License which permits any use, distribution and reproduction in any medium, provided the original author(s) and source are credited.

\section{References}

[1] ATLAS collaboration, Observation of a new particle in the search for the Standard Model Higgs boson with the ATLAS detector at the LHC, Phys. Lett. B 716 (2012) 1 [arXiv: 1207.7214] [INSPIRE].

[2] CMS collaboration, Observation of a new boson at a mass of $125 \mathrm{GeV}$ with the CMS experiment at the LHC, Phys. Lett. B 716 (2012) 30 [arXiv:1207.7235] [INSPIRE].

[3] ATLAS collaboration, Measurements of the properties of the Higgs-like boson in the four lepton decay channel with the ATLAS detector using $25 \mathrm{fb}^{-1}$ of proton-proton collision data, ATLAS-CONF-2013-013 (2013).

[4] ATLAS collaboration, Study of the spin properties of the Higgs-like particle in the

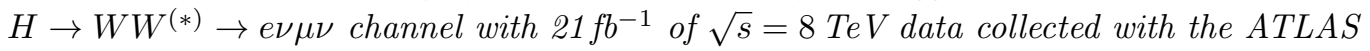
detector, ATLAS-CONF-2013-031 (2013).

[5] ATLAS collaboration, Study of the spin of the Higgs-like boson in the two photon decay channel using $20.7 \mathrm{fb}^{-1}$ of pp collisions collected at $\sqrt{s}=8 \mathrm{TeV}$ with the ATLAS detector, ATLAS-CONF-2013-029 (2013).

[6] CMS collaboration, Properties of the observed Higgs-like resonance using the diphoton channel, CMS-PAS-HIG-13-016 (2013).

[7] CMS collaboration, Study of the Mass and Spin-Parity of the Higgs Boson Candidate Via Its Decays to Z Boson Pairs, Phys. Rev. Lett. 110 (2013) 081803 [arXiv:1212.6639] [INSPIRE].

[8] ATLAS collaboration, Combined coupling measurements of the Higgs-like boson with the ATLAS detector using up to $25 \mathrm{fb}^{-1}$ of proton-proton collision data, ATLAS-CONF-2013-034 (2013).

[9] CMS collaboration, Combination of standard model Higgs boson searches and measurements of the properties of the new boson with a mass near $125 \mathrm{GeV}$, CMS-PAS-HIG-13-005 (2013).

[10] B. Patt and F. Wilczek, Higgs-field portal into hidden sectors, hep-ph/0605188 [INSPIRE].

[11] G. Bélanger, B. Dumont, U. Ellwanger, J. Gunion and S. Kraml, Global fit to Higgs signal strengths and couplings and implications for extended Higgs sectors, Phys. Rev. D 88 (2013) 075008 [arXiv: 1306 . 2941] [INSPIRE]. 
[12] J.R. Espinosa, M. Muhlleitner, C. Grojean and M. Trott, Probing for Invisible Higgs Decays with Global Fits, JHEP 09 (2012) 126 [arXiv:1205.6790] [INSPIRE].

[13] P.P. Giardino, K. Kannike, M. Raidal and A. Strumia, Is the resonance at $125 \mathrm{GeV}$ the Higgs boson?, Phys. Lett. B 718 (2012) 469 [arXiv:1207.1347] [inSPIRE].

[14] M. Montull and F. Riva, Higgs discovery: the beginning or the end of natural EWSB?, JHEP 11 (2012) 018 [arXiv:1207.1716] [INSPIRE].

[15] J. Espinosa, C. Grojean, M. Muhlleitner and M. Trott, First Glimpses at Higgs' face, JHEP 12 (2012) 045 [arXiv: 1207.1717] [INSPIRE].

[16] D. Carmi, A. Falkowski, E. Kuflik, T. Volansky and J. Zupan, Higgs After the Discovery: A Status Report, JHEP 10 (2012) 196 [arXiv:1207.1718] [INSPIRE].

[17] S. Banerjee, S. Mukhopadhyay and B. Mukhopadhyaya, New Higgs interactions and recent data from the LHC and the Tevatron, JHEP 10 (2012) 062 [arXiv:1207.3588] [INSPIRE].

[18] D. Bertolini and M. McCullough, The Social Higgs, JHEP 12 (2012) 118 [arXiv:1207.4209] [INSPIRE].

[19] K. Cheung, J.S. Lee and P.-Y. Tseng, Higgs Precision (Higgcision) Era begins, JHEP 05 (2013) 134 [arXiv:1302.3794] [INSPIRE].

[20] A. Celis, V. Ilisie and A. Pich, LHC constraints on two-Higgs doublet models, JHEP 07 (2013) 053 [arXiv: 1302.4022] [INSPIRE].

[21] G. Bélanger, B. Dumont, U. Ellwanger, J. Gunion and S. Kraml, Status of invisible Higgs decays, Phys. Lett. B 723 (2013) 340 [arXiv:1302.5694] [inSPIRE].

[22] A. Falkowski, F. Riva and A. Urbano, Higgs at last, arXiv:1303.1812 [INSPIRE].

[23] P.P. Giardino, K. Kannike, I. Masina, M. Raidal and A. Strumia, The universal Higgs fit, arXiv:1303.3570 [INSPIRE].

[24] J. Ellis and T. You, Updated Global Analysis of Higgs Couplings, JHEP 06 (2013) 103 [arXiv: 1303.3879] [INSPIRE].

[25] A. Djouadi and G. Moreau, The couplings of the Higgs boson and its CP properties from fits of the signal strengths and their ratios at the 7+8 TeV LHC, arXiv:1303.6591 [INSPIRE].

[26] A. Djouadi, O. Lebedev, Y. Mambrini and J. Quevillon, Implications of LHC searches for Higgs-portal dark matter, Phys. Lett. B 709 (2012) 65 [arXiv:1112.3299] [INSPIRE].

[27] XENON1T collaboration, E. Aprile, The XENON1T Dark Matter Search Experiment,

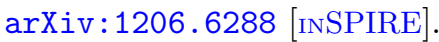

[28] XENON collaboration, M. Alfonsi, The XENON Dark Matter programme: From XENON100 to XENON1T, PoS(DSU 2012) 047 [INSPIRE]

[29] L. Lopez-Honorez, T. Schwetz and J. Zupan, Higgs portal, fermionic dark matter and a Standard Model like Higgs at 125 GeV, Phys. Lett. B 716 (2012) 179 [arXiv:1203.2064] [INSPIRE].

[30] J.F. Kamenik and C. Smith, Could a light Higgs boson illuminate the dark sector?, Phys. Rev. D 85 (2012) 093017 [arXiv: 1201.4814] [INSPIRE].

[31] XENON100 collaboration, E. Aprile et al., Dark Matter Results from 225 Live Days of XENON100 Data, Phys. Rev. Lett. 109 (2012) 181301 [arXiv:1207.5988] [INSPIRE]. 
[32] M. Beltrán, D. Hooper, E.W. Kolb and Z.C. Krusberg, Deducing the nature of dark matter from direct and indirect detection experiments in the absence of collider signatures of new physics, Phys. Rev. D 80 (2009) 043509 [arXiv:0808.3384] [InSPIRE].

[33] J.-M. Zheng et al., Constraining the interaction strength between dark matter and visible matter: I. fermionic dark matter, Nucl. Phys. B 854 (2012) 350 [arXiv:1012.2022] [INSPIRE].

[34] Z.-H. Yu et al., Constraining the interaction strength between dark matter and visible matter: II. scalar, vector and spin-3/2 dark matter, Nucl. Phys. B 860 (2012) 115 [arXiv: 1112.6052] [INSPIRE].

[35] R. Cotta, J. Hewett, M. Le and T. Rizzo, Bounds on Dark Matter Interactions with Electroweak Gauge Bosons, arXiv:1210.0525 [INSPIRE].

[36] J.-Y. Chen, E.W. Kolb and L.-T. Wang, Dark matter coupling to electroweak gauge and Higgs bosons: an effective field theory approach, arXiv:1305.0021 [INSPIRE].

[37] B. Holdom, Two U(1)'s and Epsilon Charge Shifts, Phys. Lett. B 166 (1986) 196 [InSPIRE].

[38] Planck collaboration, P. Ade et al., Planck 2013 results. I. Overview of products and scientific results, arXiv:1303.5062 [INSPIRE].

[39] Particle Data Group collaboration, J. Beringer et al., Review of Particle Physics (RPP), Phys. Rev. D 86 (2012) 010001 [inSPIRE].

[40] XENON100 collaboration, E. Aprile et al., Limits on spin-dependent WIMP-nucleon cross sections from 225 live days of XENON100 data, Phys. Rev. Lett. 111 (2013) 021301 [arXiv:1301.6620] [INSPIRE].

[41] M. Garny, A. Ibarra, M. Pato and S. Vogl, On the spin-dependent sensitivity of XENON100, Phys. Rev. D 87 (2013), no. 5056002 [arXiv:1211.4573] [INSPIRE].

[42] G. Bélanger, F. Boudjema, A. Pukhov and A. Semenov, Dark matter direct detection rate in a generic model with MicrOMEGAs 2.2, Comput. Phys. Commun. 180 (2009) 747 [arXiv:0803.2360] [INSPIRE].

[43] J. Fan, M. Reece and L.-T. Wang, Non-relativistic effective theory of dark matter direct detection, JCAP 11 (2010) 042 [arXiv:1008.1591] [INSPIRE].

[44] Fermi-LAT collaboration, M. Ackermann et al., Constraining Dark Matter Models from a Combined Analysis of Milky Way Satellites with the Fermi Large Area Telescope, Phys. Rev. Lett. 107 (2011) 241302 [arXiv:1108.3546] [INSPIRE].

[45] A. Geringer-Sameth and S.M. Koushiappas, Exclusion of canonical WIMPs by the joint analysis of Milky Way dwarfs with Fermi, Phys. Rev. Lett. 107 (2011) 241303 [arXiv: 1108.2914] [INSPIRE].

[46] X.-G. He, T. Li, X.-Q. Li, J. Tandean and H.-C. Tsai, Constraints on Scalar Dark Matter from Direct Experimental Searches, Phys. Rev. D 79 (2009) 023521 [arXiv:0811.0658] [INSPIRE].

[47] J. Kopp, V. Niro, T. Schwetz and J. Zupan, Leptophilic Dark Matter in Direct Detection Experiments and in the Sun, PoS (IDM2010) 118 [arXiv:1011.1398] [INSPIRE].

[48] R.N. Mohapatra and G. Senjanović, Neutrino Mass and Spontaneous Parity Violation, Phys. Rev. Lett. 44 (1980) 912 [INSPIRE]. 
[49] J. Schechter and J. Valle, Neutrino Masses in $\mathrm{SU}(2) \times \mathrm{U}(1)$ Theories, Phys. Rev. D 22 (1980) 2227 [INSPIRE].

[50] G. Lazarides, Q. Shafi and C. Wetterich, Proton Lifetime and Fermion Masses in an $\mathrm{SO}(10)$ Model, Nucl. Phys. B 181 (1981) 287 [InSPIRE].

[51] M. Nebot, J.F. Oliver, D. Palao and A. Santamaria, Prospects for the Zee-Babu Model at the CERN LHC and low energy experiments, Phys. Rev. D 77 (2008) 093013 [arXiv:0711.0483] [INSPIRE].

[52] MEG collaboration, J. Adam et al., New constraint on the existence of the $\mu^{+} \rightarrow e^{+} \gamma$ decay, Phys. Rev. Lett. 110 (2013) 201801 [arXiv:1303.0754] [INSPIRE].

[53] A. Akeroyd, M. Aoki and H. Sugiyama, Lepton Flavour Violating Decays $\tau \rightarrow \overline{l l l}$ and $\mu \rightarrow e \gamma$ in the Higgs Triplet Model, Phys. Rev. D 79 (2009) 113010 [arXiv:0904.3640] [InSPIRE].

[54] E.J. Chun, K.Y. Lee and S.C. Park, Testing Higgs triplet model and neutrino mass patterns, Phys. Lett. B 566 (2003) 142 [hep-ph/0304069] [INSPIRE].

[55] M. Kakizaki, Y. Ogura and F. Shima, Lepton flavor violation in the triplet Higgs model, Phys. Lett. B 566 (2003) 210 [hep-ph/0304254] [INSPIRE].

[56] CMS collaboration, A search for a doubly-charged Higgs boson in pp collisions at $\sqrt{s}=7$ TeV, Eur. Phys. J. C 72 (2012) 2189 [arXiv:1207.2666] [InSPIRE].

[57] J.F. Gunion and H.E. Haber, The CP conserving two Higgs doublet model: The Approach to the decoupling limit, Phys. Rev. D 67 (2003) 075019 [hep-ph/0207010] [INSPIRE].

[58] G. Branco et al., Theory and phenomenology of two-Higgs-doublet models, Phys. Rept. 516 (2012) 1 [arXiv:1106.0034] [INSPIRE].

[59] ATLAS collaboration, Search for invisible decays of a Higgs boson produced in association with a $Z$ boson in ATLAS, ATLAS-CONF-2013-011 (2013).

[60] LHC Higgs Cross Section Working Group collaboration, S. Heinemeyer et al., Handbook of LHC Higgs Cross Sections: 3. Higgs Properties, arXiv:1307.1347 [INSPIRE].

[61] A. Lazopoulos, T. McElmurry, K. Melnikov and F. Petriello, Next-to-leading order QCD corrections to $t \bar{t} Z$ production at the LHC, Phys. Lett. B 666 (2008) 62 [arXiv:0804.2220] [INSPIRE].

[62] A. Kardos, Z. Trócsányi and C. Papadopoulos, Top quark pair production in association with a Z-boson at NLO accuracy, Phys. Rev. D 85 (2012) 054015 [arXiv:1111.0610] [InSPIRE].

[63] M. Garzelli, A. Kardos, C. Papadopoulos and Z. Trócsányi, Z0-boson production in association with a top anti-top pair at NLO accuracy with parton shower effects, Phys. Rev. D 85 (2012) 074022 [arXiv:1111.1444] [INSPIRE].

[64] C. Englert, J. Jaeckel, E. Re and M. Spannowsky, Evasive Higgs Maneuvers at the LHC, Phys. Rev. D 85 (2012) 035008 [arXiv:1111.1719] [InSPIRE].

[65] A. Djouadi, The anatomy of electro-weak symmetry breaking. I: The Higgs boson in the standard model, Phys. Rept. 457 (2008) 1 [hep-ph/0503172] [INSPIRE].

[66] S. Dittmaier et al., Handbook of LHC Higgs Cross Sections: 2. Differential Distributions, arXiv:1201.3084 [INSPIRE].

[67] A. Abada, D. Ghaffor and S. Nasri, A Two-Singlet Model for Light Cold Dark Matter, Phys. Rev. D 83 (2011) 095021 [arXiv:1101.0365] [INSPIRE]. 
[68] A. Abada and S. Nasri, RGE of a Cold Dark Matter Two-Singlet Model, Phys. Rev. D 88 (2013) 016006 [arXiv: 1304.3917] [INSPIRE].

[69] LeP Working Group for Higgs boson searches, ALEPH, DELPHI, L3 and OPAL collaborations, R. Barate et al., Search for the standard model Higgs boson at LEP, Phys. Lett. B 565 (2003) 61 [hep-ex/0306033] [INSPIRE].

[70] V. Halyo, H.K. Lou, P. Lujan and W. Zhu, Data Driven Search in the Displaced $b \bar{b}$ Pair Channel for a Higgs Boson Decaying to Long-Lived Neutral Particles, arXiv:1308.6213 [INSPIRE].

[71] P. Gondolo and G. Gelmini, Cosmic abundances of stable particles: improved analysis, Nucl. Phys. B 360 (1991) 145 [INSPIRE].

[72] K. Griest and D. Seckel, Three exceptions in the calculation of relic abundances, Phys. Rev. D 43 (1991) 3191 [INSPIRE].

[73] B. Dumont, S. Fichet and G. von Gersdorff, A bayesian view of the Higgs sector with higher dimensional operators, JHEP 07 (2013) 065 [arXiv: 1304.3369] [INSPIRE].

[74] G. Bélanger, B. Dumont, U. Ellwanger, J. Gunion and S. Kraml, Higgs couplings at the end of 2012, JHEP 02 (2013) 053 [arXiv: 1212.5244] [INSPIRE].

[75] G. Cacciapaglia, A. Deandrea, G.D. La Rochelle and J.-B. Flament, Higgs couplings beyond the Standard Model, JHEP 03 (2013) 029 [arXiv: 1210.8120] [INSPIRE].

[76] S. Fajfer, A. Greljo, J.F. Kamenik and I. Mustac, Light Higgs and vector-like quarks without prejudice, JHEP 07 (2013) 155 [arXiv:1304.4219] [INSPIRE].

[77] ATLAS collaboration, Measurements of the properties of the Higgs-like boson in the $W W^{(*)} \rightarrow \ell \nu \ell \nu$ decay channel with the ATLAS detector using $25 \mathrm{fb}^{-1}$ of proton-proton collision data, ATLAS-CONF-2013-030 (2013).

[78] ATLAS collaboration, Measurements of the properties of the Higgs-like boson in the two photon decay channel with the ATLAS detector using $25 \mathrm{fb}^{-1}$ of proton-proton collision data, ATLAS-CONF-2013-012 (2013).

[79] P.P. Giardino, K. Kannike, M. Raidal and A. Strumia, Is the resonance at $125 \mathrm{GeV}$ the Higgs boson?, Phys. Lett. B 718 (2012) 469 [arXiv:1207.1347] [INSPIRE].

[80] CMS collaboration, Search for the standard model Higgs boson produced in association with $W$ or $Z$ bosons, and decaying to bottom quarks for LHCp 2013, CMS-PAS-HIG-13-012 (2013).

[81] CMS collaboration, Searches for Higgs bosons in pp collisions at $\sqrt{s}=7$ and $8 \mathrm{TeV}$ in the context of four-generation and fermiophobic models, Phys. Lett. B $\mathbf{7 2 5}$ (2013) 36 [arXiv: 1302.1764] [INSPIRE].

[82] CMS collaboration, Search for the standard model Higgs boson produced in association with a top-quark pair in pp collisions at the LHC, JHEP 05 (2013) 145 [arXiv:1303.0763] [INSPIRE].

[83] CMS collaboration, Properties of the Higgs-like boson in the decay $H \rightarrow Z Z \rightarrow 4 l$ in $p p$ collisions at $\sqrt{s}=7$ and $8 \mathrm{TeV}$, CMS-PAS-HIG-13-002 (2013).

[84] CMS collaboration, Updated measurements of the Higgs boson at $125 \mathrm{GeV}$ in the two photon decay channel, CMS-PAS-HIG-13-001 (2013). 